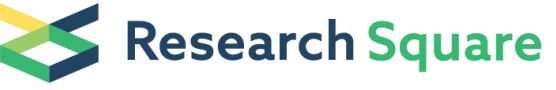

\section{Exosomal MMP-1 transfers metastasis potential in triple-negative breast cancer through PAR1- mediated EMT}

\section{Yihui Zhu}

Shanghai Cancer Hospital: Fudan University Shanghai Cancer Center https://orcid.org/0000-00025928-279X

\section{Zhonghua Tao}

Shanghai Cancer Hospital: Fudan University Shanghai Cancer Center

\section{Ying Chen}

Shanghai Cancer Hospital: Fudan University Shanghai Cancer Center

\section{Shuchen Lin}

Shanghai Cancer Hospital: Fudan University Shanghai Cancer Center

\section{Mingyu Zhu}

Shanghai Cancer Hospital: Fudan University Shanghai Cancer Center

\section{Ting Li ( $\nabla$ cinderellaliting@126.com )}

Shanghai Cancer Hospital: Fudan University Shanghai Cancer Center

\section{Xichun Hu}

Shanghai Cancer Hospital: Fudan University Shanghai Cancer Center https://orcid.org/0000-00016148-9186

\section{Research Article}

Keywords: exosomes, MMP-1, triple-negative breast cancer, metastasis, PAR1, EMT

Posted Date: August 10th, 2021

DOl: https://doi.org/10.21203/rs.3.rs-751066/v1

License: (c) (1) This work is licensed under a Creative Commons Attribution 4.0 International License. Read Full License

Version of Record: A version of this preprint was published at Breast Cancer Research and Treatment on March 7th, 2022. See the published version at https://doi.org/10.1007/s10549-022-06514-6. 


\section{Abstract \\ Purpose}

Triple-negative breast cancer (TNBC) is a subtype of breast cancer with high risk of distant metastasis, in which the intercellular communication between tumor cells also plays a role. Exosomes can be released by tumor cells and promote distant metastasis through intercellular communication or changes in tumor microenvironment, it is an optimized transportation facility for biologically active payloads. This was a hypothesis-generating research on role of exosomal payload in TNBC distant metastasis.

\section{Methods}

Exosomes isolated from supernatant of MDA-MB-231 and MDA-MB-231-HM (a highly pulmonary metastatic variant of parental MDA-MB-231 cells) were characterized. Transwell assay, wound healing and CCK-8 assay were employed to explore the effect of exosomal MMP-1 on the metastatic capability of TNBC cells in vitro. Human breast cancer lung metastasis model in nude mice was established to observe the effect of exosomal MMP-1 in vivo. Tissue microarray and blood samples of TNBC patients were applied to analyze the relevance between MMP-1 with metastasis.

\section{Results}

MDA-MB-231-HM cells secrete exosomes enriched MMP-1, which can be taken up and enhance invasion and migration activities of TNBC cells. After ingesting exosomes enriched with MMP-1, cells secrete more MMP-1, which may interact with membrane $G$ protein receptor protease activated receptor 1 (PAR1), thereby initiating epithelial-mesenchymal transition (EMT) to enhance capability of migration and invasion. The expressions of MMP-1 and PAR1 in the metastases of the 231-HM-exo treated mice were both up-regulated. Clinically, the exosomal MMP-1 can be detected from serum of patients with metastasis at higher concentration than that in pre-operative patients. Moreover, in patients with multiple distant metastases, the level of exosomal MMP-1 is also higher than that in patients with single lesion.

\section{Conclusion}

MMP-1 from TNBC cells of high metastasis potential can promote the distant metastasis of transform those with low metastasis potential through PAR1-mediated EMT and is likely to be a potential molecular marker.

\section{Background}

Breast cancer is the most commonly diagnosed malignant tumor and the sixth cause of cancer-related deaths in women ${ }^{1}$. In China, breast cancer is usually diagnosed at 45 to 55 years old, $15-20 \%$ of which is 
triple-negative breast cancer (TNBC) ${ }^{2}$. TNBC is a subtype of breast cancer with high malignancy, specifically referring to negativity of estrogen receptor (ER), progesterone receptor (PR) and human epidermal growth factor receptor-2 (HER-2). TNBC is characterized by a high risk of recurrence and metastasis, a short progression-free survival (PFS) and overall survival (OS) ${ }^{3}$. TNBC also has a tendency to metastasize to different organs including the lung, liver, brain and bones, leading to different prognosis and response to treatment ${ }^{4}$. Regarding the mechanism of breast cancer metastasis, most prior studies have focused on the process of metastasis. The interaction between cancer cell clones was less to be explored in terms of teamwork in the formation of distant metastasis.

Exosome is a subtype of extracellular vesicles (EV) with a diameter of 30 to $150 \mathrm{~nm}$, which can be secreted by almost all cells and detected in body fluids such as blood, saliva, cerebrospinal fluid, urine, semen and tumor microenvironment ${ }^{5}$. Exosomes carry proteins, lipids, mRNA and circRNA etc., which are considered to play an important role in tumor development such as tumor growth, invasion, metastasis, and regulation of tumor microenvironment ${ }^{6}$. There is increasing evidence showing that exosomes play an important role in tumor spread ${ }^{7}$ (for example, establishing a pre-metastasis niche ${ }^{8}$, promoting angiogenesis ${ }^{9}$, destroying the peritoneum ${ }^{10}$ or the blood-brain barrier ${ }^{11}$ ), inducing drug resistance ${ }^{12}$ and producing cancer-related fibroblast heterogeneity ${ }^{13}$. We speculate that cells with high metastasis ability can deliver metastasis-promoting substances to cells with normal metastasis ability through exosomes, initiate a series of downstream cascade reactions to enhance their metastasis ability. Our experiments in vitro showed that after co-cultivation of exosomes derived from classic TNBC breast cancer cells (MDAMB-231) and exosomes derived from high lung metastasis breast cancer cells built in our laboratory (MDA-MB-231-HM) ${ }^{14-16}$, the invasion capability of MDA-MB-231 was significantly improved. Therefore, we speculate that the key protein carried in exosomes derived from highly metastatic cell lines is one of the relevant factors that promote invasion and distant metastasis. This study used mass spectrometry to screen the differential proteins in exosomes derived from the high lung-metastatic cell line MDA-MB-231HM and the classic TNBC cell line MDA-MB-231, found MMP-1 to be the most different protein. MMPs are a family of zinc-dependent endopeptidases which are crucial to ECM degradation. Several MMPs have been detected in exosomes from tumor cells ${ }^{17}$. MT1-MMP existing in exosomes from tumor cells is involved in activation of proMMP-2 as well as degradation of ECM proteins ${ }^{18,19}$. It was also reported that combined expression of miR-21 and MMP-1 in urinary exosomes detects $95 \%$ of breast cancer ${ }^{20}$. We suppose that MMP-1 may be a key protein to mediate the transfer of metastatic ability between tumor cells through exosomes.

\section{Methods}

\section{Tissue samples}

Tissue specimens $(n=134)$ were collected from female patients with TNBC, defined as ER negative, PR negative, HER2 negative or 1+ or 2+ but fluorescence in situ hybridization (FISH) negative, who underwent operation of tumor in Fudan University Shanghai Cancer Center (Shanghai, China) between August of 
2015 and May of 2016. After selection of formalin-fixed paraffin embedded (FFPE) tissue blocks of tumor sections, 5 tissue microarrays (TMA) blocks with 50 cores per TMA were constructed. Sections were cut at $4 \mu \mathrm{M}$ thick for immunohistochemistry. Clinical characteristics were obtained from the Electronic Medical Record System and telephone follow-up. The research protocol was approved by the Ethics Committee of the Fudan University Shanghai Cancer Center, and written informed consent was obtained from all patients prior to enrollment.

\section{Immunohistochemical staining}

Standard immunohistochemical procedures were carried out using anti-MMP-1(26585-1-AP, Proteintech), anti-PAR1 (26366-1-AP, Proteintech) in human breast cancer TMAs and lung tumor sections of nude mice. The TMAs were digitized via whole-slide scanning with the Aperio T2 scanner (Aperio Technologies) and IOD values were assessed independently by two pathologists in from the Department of Pathology (Fudan University Shanghai Cancer Center); discrepancies were resolved by discussion until a consensus was reached. Lung tumor sections of nude mice were observed and imaged by microscope (OLYMPUS, BX43).

\section{Cell lines and culture}

MDA-MB-231, MDA-MB-468 and BT549 (human breast cancer cell line) were acquired from the Shanghai Cell Bank of Chinese Academy of Sciences (Shanghai, China). MDA-MB-231-HM cell line and was established from MDA-MB-231 using an in vivo stepwise selection scheme in Fudan University Shanghai Cancer Center (Shanghai, China), has a high potential for lung metastasis ${ }^{14}$. MDA-MB-231, MDA-MB-468 and MDA-MB-231-HM were grown in Leibovitz's L-15 medium, BT549 was cultured in RPMI 1640, containing $10 \%$ fetal bovine serum (FBS), penicillin $(100 \mathrm{U} / \mathrm{ml})$, and streptomycin $(100 \mathrm{mg} / \mathrm{ml})$ in incubator at $37^{\circ} \mathrm{C}$ (both Gibco; Thermo Fisher Scientific, Inc., Waltham, MA, USA).

\section{Tumor cell migration and invasion assays}

24-well plates inserted with 8-mm pore size filters (Corning Life Sciences, Corning, NY, USA), for tumor cell invasion ability, the filter membranes were pre-coated with 20 $\mu$ l Matrigel (dilution, 1:5; BD Biosciences, Franklin Lakes, NJ, USA). Cells were seeded into the upper chambers, with L-15 containing $20 \%$ FBS in the lower chambers. Cells were incubated for $24-36 \mathrm{~h}$ at $37^{\circ} \mathrm{C}$, and cells that had invaded or migrated to the reverse side of the membrane were detected by staining with crystal violet, viewed and counted in at least five random fields under a light microscope (OLYMPUS, IX51).

\section{Cell proliferation}

A Cell Counting Kit-8 (PN812; Dojindo, Japan) was used for cell proliferation assays. Cells were seeded into a 96 -well plate at a density of $2 \times 10^{3}$ cells per well in quintuplicate wells. At day 1-6 after culture, $10 \mu \mathrm{l}$ of CCK-8 solution was added to each well and incubated for $2 \mathrm{~h}$ at $37^{\circ} \mathrm{C}$, the absorbance of cells was 
measured at a wavelength of $450 \mathrm{~nm}$ for calculation of the optical density (OD) values (Synergy H1, Biotek).

\section{Wound healing assay}

Cells were seeded in 6-well plates and grew to almost complete confluence, then a $10 \mu$ pipette tip was used to scratch a gap on the cell layer. Cells were incubated in serum-free medium. The gap widths were measured and imaged under an inverted microscope (OLYMPUS, IX51) at 0h, 24h and $36 \mathrm{~h}$ after scratching.

\section{Purification of exosomes from cell and human plasma}

When cells grew at about $40-50 \%$ confluence, they were moved to medium containing exosome-depleted FBS, which was obtained by ultracentrifugation of standard FBS at $100,000 \mathrm{~g}$ for $10 \mathrm{~h}$ at $4^{\circ} \mathrm{C}$ followed by filtration through a $0.22 \mu \mathrm{m}$ vacuum filtration bottle. Supernatants were collected from $24-36 \mathrm{~h}$ cell cultures. Exosomes were purified by differential centrifugation at $3,000 \mathrm{~g}$ for $20 \mathrm{~min}$ to remove cell debris and dead cells. Vesicles were pelleted after centrifugation at $16,500 \mathrm{~g}$ for $45 \mathrm{~min}$ and resuspended in PBS, then centrifuged at $100,000 \mathrm{~g}$ for $2 \mathrm{~h}$ at $4^{\circ} \mathrm{C}$ (Beckman Coulter, Optima XPN-100). After resuspending in an appropriate amount of PBS, the protein concentration was measured, recorded and stored for subsequent experiments.

For purification of circulating exosomes by differential centrifugation, blood from patients was centrifuged at $1,500 \mathrm{~g}$ for $30 \mathrm{~min}$ to obtain cell-free plasma. Plasma was centrifuged at $15,000 \mathrm{~g}$ for $30 \mathrm{~min}$. The pelleted vesicles were suspended in PBS and then centrifuged at $100,000 \mathrm{~g}$ for $2 \mathrm{~h}$ at $4{ }^{\circ} \mathrm{C}$.

\section{Transmission electron microscopy (TEM) and Nanosight analysis (NTA) of exosomes}

Purified exosomes were prepared on copper TEM grids (3.05mm; 200 mesh) by negative staining. 10 $\mu$ l sample was dropped on copper grids and incubated for 3 mins. $10 \mu \mathrm{l} 2 \%$ uranyl acetate was pipetted on the grid and incubated for $10 \mathrm{~min}$. Excess solution was removed by with filter paper. Grid was stored in the dark grid box at RT until imaging. Imaging was conducted using a 120kV Biology Transmission Electron Microscope (Tecnai G2 SpiritBiotwin).

Nanoparticle tracking analysis was conducted by NanoSight NS300. Exosomes in 20 $\mu$ I PBS were diluted to $1000 \mu \mathrm{l}$ with DPBS, then loaded with a $1 \mathrm{ml}$ syringe to be tested. Three recordings of $30 \mathrm{~s}$ at $37^{\circ} \mathrm{C}$ in camera were obtained and processed using NTA software.

\section{Exosome labeling}

Exosomes were resuspended in PBS and stained with DIO dye for $20 \mathrm{~min}$ at room temperature. The labeled exosomes were incubated with MDA-MB-231 cells pre-seeded on the small discs with medium containing exosome-depleted FBS for $24 \mathrm{~h}$ at $37^{\circ} \mathrm{C}$. Then the cells were washed three times in PBS-T, fixed with $4 \%$ paraformaldehyde for $15 \mathrm{~min}$ at room temperature, then washed 3 times with PBS-T. The cell 
nuclei were counterstained with $5 \mathrm{ug} / \mathrm{ml}$ DAPI (Cat.0100-20, SouthernBiotech) for $5 \mathrm{~min}$. The uptake of the labeled exosomes by MDA-MB-231 cells was assessed using an inverted confocal microscope (Leica TCS SP8, Germany).

\section{Enzyme-linked immunosorbent assay (ELISA)}

100ul patient serum, exosomes purified from $250 \mu$ patient serum and $10 \mathrm{ml}$ cell culture media suspended in 100 $\mu$ l PBS were transferred to a clean tube. The concentrations of MMP-1 were determined using ELISA kits (Cat. EHC134, Quanticyto, Neobiscience, Inc, Shenzhen, China) according to the manufacturer's protocols.

\section{Construction and transfection of MMP-1 short hairpin RNA (shRNA) lentivirus into tumor cells}

An MMP-1 shRNA lentivirus was purchased (HanyinBiotech, Ltd., Shanghai, China) to knock down the expression of MMP-1 in MDA-MB-231-HM cells. The target sequences against MMP-1 were 5'GCCTTCCAACTCTGGAGTAAT-3' $₫ 5$ '-GCTAGCTCAGGATGACATTGA-3' and 5'-GATTCTACATGCGCACAAATC3'. MDA-MB-231-HM cells were plated into a 6 -well plate, grown to reach $60-80 \%$ confluence and then transfected with viral supernatant diluted 1:1000 (lentivirus) in the presence of $10 \mu \mathrm{g} / \mathrm{ml}$ polybrene for $24 \mathrm{~h}$, according to the manufacturer's protocol. Cells were selected with $3 \mathrm{~g} / \mathrm{ml}$ puromycin for 7 days since $48 \mathrm{~h}$ after transduction.

\section{Western blot}

Cell and exosome samples were lysed using RIPA buffer (Beyotime, Shanghai, China) containing protease and phosphatase inhibitors (Epizyme, Shanghai, China). The protein concentration was quantified using a BCA protein assay kit (Beyotime). Proteins were separated by $10 \%$ (gradient) SDS-PAGE (Epizyme) and

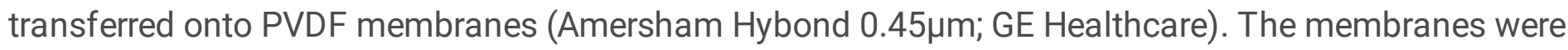
blocked with $5 \%$ milk in trisbuffered saline-Tween (TBS-T) for $2 \mathrm{~h}$, then incubated with primary antibodies (anti-CD9(ab92726,Abcam), anti-CD63(ab134045,Abcam), anti-MMP-1(ab134184,Abcam), antiPAR1(26366-1-AP,Proteintech), anti-Zeb-1 (21544-1-AP,Proteintech), anti-Slug(12129-1-AP,Proteintech), antiSNAI1(13099-1-AP,Proteintech), anti-E-cadherin(20874-1-AP,Proteintech), anti-Vimentin(10366-1AP,Proteintech), anti-GAPDH(60004-1-Ig,Proteintech)) for $12 \mathrm{~h}$ at $4^{\circ} \mathrm{C}$. the membranes were washed with TBS-T for 3times, $15 \mathrm{~min}$ per time, then incubated with horseradish peroxidase (HRP)-conjugated secondary antibodies for $1 \mathrm{~h}$ at RT. The proteins were visualized using the ECL western blotting substrate (cat. WBKLS0100, Millipore) and a Tanon-5200Multi device.

\section{Lung colonization study}

Six- to eight-week-old nude mice were injected in tail vein with $1 \times 10^{6}$ MDA-MB-231 cells. Cell-derived exosomes $(15 \mu \mathrm{g}$ in $100 \mu \mathrm{l}$ PBS) were intravenous injected every other day for 2 weeks. The mice were euthanized six weeks after the cancer cell injection and their lungs were fixed, sectioned and analyzed for H\&E quantify the metastatic tumor burden. Paraformaldehyde-fixed lungs were sectioned into 8- $\mu \mathrm{m}$-thick 
sections, respectively, at $100 \mu \mathrm{m}$ intervals. Three large lung sections were stained with H\&E and tumor nodules were counted and their area measured using the OlyVIA (Olympus) and ImageJ software. The number of the metastatic nodules were calculated by averaging data from individual sections.

\section{Co-immunoprecipitation}

Co-immunoprecipitation of MMP-1 or PAR1 from MDA-MB-231 cell lysates was carried out as follow. MMP-1 and PAR1 were immunoprecipitated using a rabbit anti-MMP-1 or anti-PAR1 antibody. Rabbit IgG were used as negative controls. Immune complexes were captured using the Protein A/G-agarose beads (LOT. 70474621, Roche), and immunoprecipitates were analyzed by SDS-PAGE followed by immunoblotting with rabbit monoclonal anti-MMP-1 antibody (26585-1-AP, Proteintech) or anti-PAR1 antibody (26366-1-AP, Proteintech). Immunoreactive bands were visualized using enhanced chemiluminescence.

\section{Chemicals and inhibitors}

Vorapaxar (HY-10119) was obtained from MedChemExpress.

\section{Statistical analysis}

All experiments in the study were performed in triplicate. Statistical analyses were performed with the SPSS v20.0 software (SPSS Inc., Chicago, IL, USA). Data are presented as the mean \pm standard deviation (SD). Quantitative data were compared with a two-tailed Student's t-test between groups and a one-way analysis of variance among multiple groups followed by Lease Significant Difference post hoc test. Kaplan-Meier curves of disease-free survival were plotted and survival in the groups was compared by log-rank test. The significance levels of the data are denoted by * symbols as follows: ${ }^{*} p<0.05 ;{ }^{* \star} p<0.01$; $\star \star \star x p 0.001$; and ${ }^{* \star \star \star} p<0.0001$. $p<0.05$ was considered to indicate a statistically significant difference.

\section{Bioinformatics analysis}

The association between MMP1 expression and prognosis in triple-negative breast cancer patients was analyzed by using the Kaplan Meier plotter, which is an online database that provides assessment of the effect of 54,675 genes on survival using 10,293 cancer samples, including 22,277 genes in 5,143 breast cancer samples (http://kmplot.com/analysis/).

\section{Results}

\section{Isolation and verification of exosomes}

In our previously study, MDA-MB-231-HM (MDA-231-HM), a highly pulmonary metastatic variant of parental MDA-MB-231 (MDA-231) cells, was derived within the presented model system by six cycles of the pulmonary metastasis implantation to the mammary fat pad (MFP) ${ }^{14}$, which was a valuable model system for the study of the molecular events underlying breast cancer metastasis ${ }^{15,16}$. The differences in 
pulmonary metastatic potential of the two cell lines make them valuable systems for understanding the molecular mechanisms underlying breast cancer metastasis. Transwell assay showed that migration and invasion capabilities of MDA-231-HM are stronger than MDA-231 cells (Figure 1A).

We purified exosomes from supernatants of MDA-231 and MDA-231-HM cells by differential centrifugation ${ }^{21,22}$, and verified them by Transmission electron microscopy (TEM) (Figure 1B), Western blot (Figure 1C) and nanoparticle tracking analysis (NTA) (Figure 1D). After co-cultivating MDA-231 cells with 231-HM-exo for 24h, the exosomes were observed by confocal microscopy close to the nucleus, indicating that the exosomes have been taken up by MDA-231 cells (Figure 1E).

\section{MMP-1 in exosomes mediates the transmission of metastasis capability}

MDA-231 cells were pretreated with PBS, 231-exo and 231-HM-exo for 24h and then analyzed by Transwell migration and invasion assay. The results showed that the migration and invasion ability of MDA-231 cells co-cultured with 231-HM-exo was significantly improved while the invasion ability of MDA231 cells co-cultured with 231-exo was improved (Figure 2A). We assume that 231-HM-exo was taken up by MDA-231 cells, releasing its cargo to increase the content of protein or RNA, which directly or indirectly activated downstream signaling pathways and improved the invasion and migration capability of MDA231 cells in vitro. In order to find the key protein transferred by exosomes that changes the ability of invasion and migration capability, we screened the differential proteins between 231-exo and 231-HM-exo by mass spectrometry. From the heat map we found that MMP-1 is the protein with the most significant difference in expression (Figure 2B).

MMP-1 belongs to matrix metalloproteinase (MMP) family, which includes a series of zinc- and calciumdependent endopeptidases. MMPs are of crucial importance for invasive cancer cells to break extracellular matrix (ECM) barriers and start the metastatic cascade ${ }^{23,24}$. MMP1 has also been shown to be related to invasive phenotype, metastasis and response to chemotherapy in human breast cancer, and is related to poor prognosis ${ }^{25-27}$. We verified the results of mass spectrometry by western blot of MMP-1 in MDA-231, MDA-231-HM, 231-exo and 231-HM-exo and saw significant difference in both cells and exosomes (Figure $2 \mathrm{C}$ ).

\section{Targeted knockdown of MMP-1 RNA inhibits metastasis through exosomes}

We used lentivirus packaged short-hairpin RNA (shRNA) to infect MDA-231-HM to knockdown MMP-1. We selected 231-HM-shRNA3, whose MMP-1 in the exosome was significantly downregulated, hereinafter referred to as MDA-231-HM-shMMP1 (Figure 3A, B). In order to rule out the change of proliferation capability caused by MMP-1, which may interfere the results of migration and invasion assays, we used CCK-8 kit to analyze the proliferation of MDA-231, MDA-231-HM, MDA-231-HM Negative Control (MDA231-HM-NC), and MDA-231-HM-shMMP1. Results showed no correlation between MMP-1 expression and cell proliferation (Figure $3 \mathrm{C}$ ). Both Transwell and wound healing assay showed that migration and invasion activity of MDA-231-HM was significantly weaken after targeted knockdown of MMP-1 (Figure 3D, E). 
After observing the differences in migration and invasion ability of cells, we focused on cell-to-cell transmission of MMP-1 through exosomes. Transwell assay showed that co-cultivation of MDA-231 with 231-HM-shMMP1-exo for 24h significantly reduced the migration and invasion of 231 cells co-cultured with 231-HM-exo. Considering the heterogeneity of TNBC, we added MDA-MB-468 (MDA-468) and BT549 to repeat the above experiment and the results were similar, proving that the role of MMP-1 in exosomes is not limited to MDA-231 cell line (Figure 3F, G).

\section{MMP-1 binding to PAR1 promotes the metastasis of TNBC possibly through EMT}

The function of MMP-1 on ECM degradation has been reported ${ }^{23,24}$, which cannot fully explain the enhanced invasion and migration activity both. MMP-1/PAR1 as a key signal to activate downstream signaling pathways to facilitate vascular intravasation and metastatic dissemination has also been proposed in several tumors ${ }^{28-30}$. Protease-activated receptor 1 (PAR1) is a G protein-coupled receptor that is classically activated by the serine protease thrombin cleavage of the $\mathrm{N}$-terminal outer domain, can also be directly activated by MMP-1 and MMP-9 ${ }^{31}$. PAR1 is also considered as an independent factor for the poor prognosis of tumors and a potential therapeutic target ${ }^{32}$.

We found that after ingestion of exosomes with different levels of MMP-1, the level of free MMP-1 secreted by MDA-231 cells into the supernatant was positively correlated with the level of MMP-1 in exosomes (Figure 4A). Then, we demonstrated through co-immunoprecipitation that MMP-1 can directly interact with PAR1 to stimulate downstream metastasis related pathways (Figure 4B). The PAR1 irreversible antagonist, Vorapaxar, was added at a concentration gradient to the medium of 231 cells cocultured with 231-HM-exo and could effectively reverse the effect of 231-HM-exo. Vorapaxar inhibited $58 \%$ and $79 \%$ of the migration, $45 \%$ and $57 \%$ of the invasion at $75 \mu \mathrm{M}$ and $100 \mu \mathrm{M}$, respectively (Figure 4C). This strongly explains that MMP-1 can not only degrade collagen to promote invasion, but mainly rely on PAR1 to exert its invasion effect. Inhibiting PAR1 can greatly inhibit this acquired capability of invasion. In order to eliminate that the change in cell proliferation ability brought by PAR1 may interfere the results of migration and invasion assays, we first used CCK-8 assay to detect the proliferation of MDA-231 cells co-cultured with 231-HM-exo and Vorapaxar. Results showed that cell proliferation was independent of 231-HM-exo and Vorapaxar (Figure 4D).

According to previous reports, exosome-associated MMPs are involved in the processes of EMT in some tumors $^{33-35}$. The decrease in keratin filaments and the decrease of E-cadherin, as well as the increase of vimentin, fibronectin, $\mathrm{N}$-cadherin, $\mathrm{a}-\mathrm{SMA}$ and various proteases, resulted in the loss of polarity and acquisition of the mesenchymal phenotype ${ }^{36,37}$. We speculated that PAR1 was activated by MMP-1 and mediated epithelial-mesenchymal transition (EMT) which enhanced the ability of metastasis in TNBC. MDA-231 cells were treated with PBS, 231-HM-exo, 231-HM-shMMP1-exo and sufficient Vorapaxar and Western blot was used to detect the expression of EMT biomarkers. The down-regulation of E-cadherin and up-regulation of ZEB-1, Vimentin and Slug in MDA-231 cells co-cultured with 231-HM-exo suggested mesenchymal phenotype, while in 231-HM-exo and Vorapaxar group, E-cadherin was up-regulated, ZEB-1, Vimentin and Slug were significantly down-regulated, suggesting epithelial phenotype. It can be seen that 
the EMT process promoted by 231-HM-exo can be reversed by PAR1 antagonist (Figure 4E). Based on the results of in vitro experiments, we propose that after 231-HM-exo is ingested by targeted cells, the secretion of MMP-1 increases, which can directly degrade ECM, and may possibly also cleave and activate PAR1-mediated EMT to promote cell migration and invasion.

\section{Exosomal MMP-1 mediates the transmission of metastasis capability in vivo.}

To verify the metastasis ability of MDA-231 and MDA-231-HM cells in vivo, we established a human breast cancer lung metastasis model in nude mice. We observed that mice injected with MDA-231-HM cells $(n=3)$ had significantly more lung metastases than MDA-231 cells $(n=3, p<0.05)$ (Figure 5A), which means that the metastatic ability of MDA-231-HM in vivo is much stronger than that of MDA-231 cells.

To determine the effect of MMP-1 in exosomes on the transfer ability of MDA-231 cells in vivo, We further constructed nude mouse lung metastasis model with MDA-231 cells and treated it with PBS, 231-exo, 231-HM-exo and 231-HM-shMMP1-exo every other day for 2 weeks (Figure 5B). Metastatic nodules in mice treated with $231 \mathrm{HM}$-exo $(n=9)$ was significantly more than that with PBS $(n=9)$ and $231-e x o(n=9)$ $(p<0.001)$, and which were reduced by 231-HM-shMMP1-exo $(n=9)(p<0.005)$ (Figure 5C-E). A hypothesis consistent with results in vitro can be drawn, exosomes with high levels of MMP-1 can promote metastasis ability of MDA-231 in vivo, which would be weaken by reducing MMP-1 in exosomes.

Conducting immunohistochemical staining for MMP-1 and PAR1 on lung lesions of nude mice, we saw the expression of MMP-1 and PAR1 in lung lesions of mice treated with 231-HM-exo increased, while the expression of 231-HM-shMMP1-exo treated group decreased (Figure 5F).

\section{Plasma exosomal MMP-1 level is elevated in patients with shorter DFS.}

A total of 134 surgical tissues from TNBC patients from August 2015 to May 2016 were collected and made into tissue microarrays (TMA). The characteristics of patients at baseline are shown in Table 1. The patients were followed up to 2020.8, median follow-up time was 56.8 months. MMP-1 expression was determined by immunohistochemistry and a quarter one fourth of the OD value of MMP-1 (OD=4.009) was chosen as a cut-off value to define 'high' and 'low' expression (Figure 6A). In the univariate KaplanMeier analysis, TNBC showing high expression of MMP-1 had a significantly shorter disease-free survival (DFS) (logrank p=0.028) (Figure 6B). Survival curve retrieved from Kaplan-Meier plotter showed that high level MMP-1 expression is associated with significantly worse DFS (logrank $p=6 e-13$ ) in TNBC patients (Figure 6C).

Blood samples from patients at baseline of pre-surgery $(n=18)$ and initial metastasis $(n=30)$, baseline characteristics are shown in Table 2. The MMP-1 expression level in serum and exosomes purified from serum was detected by ELISA. We found that MMP-1 expression in initial metastasis group was significantly higher than that in pre-surgery group, whether in serum $(p<0.0001)$ or exosomes $(p<0.0001)$. It was confirmed that the MMP-1 carried in exosomes was related to the recurrence and systemic metastasis of TNBC (Figure 6D, E). 
Furthermore, we distinguished patients with single metastatic site $(n=13)$ and multiple metastatic sites $(n=17)$ in the initial metastasis group to research the correlation between MMP-1 and systemic metastasis. The concentration of MMP- 1 in the serum has no statistical difference $(p=0.1488)$ while in exosomes, MMP-1 detected in patients with multiple metastases was significantly higher than that in single metastatic site $(p<0.01)$, which suggests that for patients with distant recurrence and metastasis, MMP-1 detected in exosomes would be more sensitive than in serum (Figure 6F, G).

Interestingly, we collected blood samples from several patients $(n=11)$ before and after first-line chemotherapy and tumor response was evaluated in accordance with the Response Evaluation Criteria in Solid Tumors (RECIST 1.1) guidelines by computed tomography scanning or magnetic resonance imaging after $2 \mathrm{cycles}^{38}$.We found that MMP-1 level in exosomes was also related to response to chemotherapy. The expression of MMP-1 in exosomes of patients with partial response (PR) $(n=4)$ decreased while increased in patients with progressive disease $(P D)(n=3)$. In patients with stable disease $(S D)(n=4)$, the expression of MMP-1 in exosomes both increased and decreased, which suggests that MMP-1 in exosomes even has the potential to be an indicator of therapeutic efficacy (Figure $6 \mathrm{H}$ ).

\section{Discussion}

Many reports demonstrated that exosomes can mediate tumor metastasis by horizontal transfer of bioactive molecules to recipient cells ${ }^{8,11,38-40}$. In this study, we first reported that TNBC cells with high metastasis potential transform those with low metastasis potential via exosomal MMP-1.

Exosomes have been reported to take part in promoting tumor metastasis and drug resistance $\mathrm{e}^{41,42}$. Exosomes potentially promote tumor development by regulating biological functions, including angiogenesis, immunity, vascular leakiness, and reprogramming recipient cells to construct premetastatic niche $(\mathrm{PMN})$ and metastasis ${ }^{43-45}$. To study the role of exosomes in tumor metastasis, we extracted, verified and observed the exosomes taken up by MDA-231 cells. Then mass spectrometry was applied for the most different expressed protein between high and low lung metastasis cell-derived exosomes, which is MMP-1.

Historically, MMPs have been studied for a long time in cancer biology, and accumulated evidence showed that MMPs are related to progression, metastasis, treatment and prognosis of cancer ${ }^{46-48}$. As reported, MMPs mediate the degradation of ECM and basement membrane (BM) at all stages of cancer progression, thereby promoting the development of the surrounding microenvironment and distant metastasis ${ }^{13,28}$. However, the fact that MMP-1 is transmitted between cells through exosomes to enhance metastasis ability of low-metastatic cells has not yet been reported.

Unsurprisingly, our study found that knocking down the MMP-1 of MDA-231-HM with shRNA reduced MMP-1 in MDA-231-HM-secreted exosomes. Hereafter, we observed that exosomes with reduced MMP-1 content actually affected the invasion and migration ability of MDA-231 in vitro and in vivo. Further, we explored the mechanism of the influence of exosomal MMP-1.The most widely known function of MMP-1 
is to degrade collagen I, II and III in the ECM, but it cannot fully explain the enhanced activity of both invasion and migration. In addition to degrading collagen, MMP-1 can also directly PAR 1 by cutting the extracellular $\mathrm{N}$-terminus of PAR $1^{26}$. PAR1 is a $\mathrm{G}$ protein-coupled receptor that is classically activated by the serine protease thrombin cleavage of the N-terminal outer domain. PAR1 activated by MMP-1 provides a link between the extracellular proteolytic activity important for ECM remodeling and the signal transduction leading to cell migration and invasion ${ }^{31}$. It was reported that MMP-1 and MMP-13 cut the Nterminal outer domain of PAR1 at non-canonical sites, activating the G-protein signaling pathway ${ }^{39}$. In breast cancer cells, thrombin and MMP-1 activated PAR1-dependent phospho-Akt signals ${ }^{46}$. In our study, immunoprecipitation assay showed that MMP-1 directly interacts with PAR1. The PAR1 antagonist, Vorapaxar can also significantly reverse the up-regulated metastatic capability of MDA-231 after coculture with 231-HM-exo at a certain concentration. It can also be seen that the EMT process promoted by 231-HM-exo can be reversed by PAR1 antagonist. We took the lung tissues for immunohistochemistry and found that the expression of MMP-1 and PAR1 were higher in the 231-HM-exo-treated group, whereas those were down-regulated with 231-HM-shMMP1-exo. Therefore, it is concluded that free MMP-1 in the supernatant, which sources may be from released MMP-1 from internalized exosomes and production by initial low metastasis capacity MDA-231 cells, binds to the membrane surface receptor PAR1 protein and then promotes the metastasis of the triple-negative breast cancer possibly through EMT.

By searching the Kaplan-Meier database, we observed that the expression level of MMP-1 is related to DFS of TNBC, and this finding was then confirmed by TMA of surgical tissue samples of TNBC patients in our center. There was also significantly up-regulated level of MMP-1 in serum and exosomes in patients with metastasis. Moreover, whereas there was no statistical difference in serum MMP-1 expression in patients with solitary lesion and multiple distant metastasis, MMP-1 in exosomes produced statistical differences, indicating that exosomes may be more sensitive and accurate in predicting and evaluating distant metastases. Furthermore, comparing exosomal MMP-1 level before and after first-line chemotherapy in several patients showed that MMP-1 alteration is associated with the efficacy, indicating implication in predicting the efficacy of chemotherapy.

There are still some limitations in this study. In terms of experimental data, first of all, due to the limitations of laboratory facilities, we were unable to record the trajectory of cell migration and draw trajectory diagrams to visually display and compare the metastatic ability of cells. Secondly, in the study of the relationship between the changes in the level of MMP-1 in exosomes and the efficacy of first-line chemotherapy in clinical patients, the number of blood samples was too small to draw a statistical conclusion. In terms of conclusion, the mechanism of EMT caused by the interaction between PAR1 and MMP-1 has not been directly clarified through experiments. We could only suppose and prove that the cells go through EMT by western blot assay for changes in EMT-related markers before and after the treatment of exosomes. Finally, the research is currently aimed at TNBC, and ER-positive and HER-2positive breast cancer cell lines may be included for further study.

\section{Conclusions}


In summary, exosomes secreted by cells of high metastasis ability enriched in MMP-1 can transform cells with low metastasis ability in TNBC into more malignant cells, which is possibly mediated EMT via interaction with PAR1. Clinically, higher MMP-1 level in exosomes in patient's blood indicates more chance of occurrence of distant metastasis, along with a certain potential for the evaluation of therapeutic effects.

\section{Abbreviations}

TNBC, Triple-negative breast cancer; EV, extracellular vesicles; MMP-1, matrix metalloproteinase-1; PAR1, protease-activated receptor 1; ER, estrogen receptor; PR, progesterone receptor; HER-2, human epidermal growth factor receptor-2; PFS, progression-free survival; OS, overall survival; FFPE, formalin-fixed paraffin embedded; TMA, tissue microarrays; OD, optical density; TEM, Transmission electron microscopy; NTA, Nanosight analysis; MFP, mammary fat pad; shRNA, short-hairpin RNA; ECM, extracellular matrix; EMT, epithelial-mesenchymal transition; DFS, disease-free survival; RECIST 1.1, Response Evaluation Criteria in Solid Tumors; PR, partial response; PD, progressive disease; SD, stable disease; PMN, pre-metastatic niche.

\section{Declarations}

\section{Funding}

This work was supported by the National Science and Technology Major Project (2020ZX09201-013) and National Natural Science Foundation of China (81702970). The sponsors were not involved in study design, experiment conduction and data analysis.

\section{Competing interests}

The authors declare that they have no competing interests.

\section{Availability of data and materials}

The datasets generated during and/or analyzed during the current study are available from the corresponding author on reasonable request.

\section{Author Contributions}

YH ZHU and ZH TAO have contributed equally to this work and share first authorship.

$\mathrm{XC} \mathrm{Hu}$ and $\mathrm{T}$ Li conceived the study and gathered together the team. YH Zhu performed the experiments. $\mathrm{YH}$ Zhu and ZH Tao contributed to experimental development control and validation, as well as to data analysis. T Li, Y Chen, SC Lin and MY Zhu provided resources for study development and data acquisition. YH Zhu prepared the figures and the original draft. All authors provided critical feedback and reviewed and edited the manuscript. XC Hu and T Li obtained the funding for the study accomplishment. 


\section{Ethics approval}

This study was approved by the Ethics Committee of Shanghai Cancer Center of Fudan University.

\section{Consent to participate}

All patients signed the informed consent.

\section{Consent for publication}

All authors are consent for publication.

\section{References}

1. Bray F, Ferlay J, Soerjomataram I, Siegel RL, Torre LA, Jemal A (2018) Global cancer statistics 2018: GLOBOCAN estimates of incidence and mortality worldwide for 36 cancers in 185 countries. CA: a cancer journal for clinicians Nov 68(6):394-424. doi:10.3322/caac.21492

2. Lee KL, Kuo YC, Ho YS, Huang YH. Triple-Negative Breast Cancer: Current Understanding and Future Therapeutic Breakthrough Targeting Cancer Stemness. Cancers Sep 9 2019;11(9)doi:10.3390/cancers11091334

3. Mustacchi G, De Laurentiis M (2015) The role of taxanes in triple-negative breast cancer: literature review. Drug Des Devel Ther 9:4303-4318. doi:10.2147/dddt.S86105

4. Liang Y, Zhang H, Song X, Yang Q (2020) Metastatic heterogeneity of breast cancer: Molecular mechanism and potential therapeutic targets. Seminars in cancer biology Feb 60:14-27. doi:10.1016/j.semcancer.2019.08.012

5. Yáñez-Mó M, Siljander PR, Andreu Z et al (2015) Biological properties of extracellular vesicles and their physiological functions. Journal of extracellular vesicles 4:27066. doi:10.3402/jev.v4.27066

6. Becker A, Thakur BK, Weiss JM, Kim HS, Peinado H, Lyden D (2016) Extracellular Vesicles in Cancer: Cell-to-Cell Mediators of Metastasis. Cancer cel/ Dec 12(6):836-848. doi:10.1016/j.ccell.2016.10.009 $30)$.

7. Naito Y, Yoshioka Y, Yamamoto Y, Ochiya T (2017) How cancer cells dictate their microenvironment: present roles of extracellular vesicles. Cellular molecular life sciences: CMLSFeb 74(4):697-713. doi:10.1007/s00018-016-2346-3

8. Deep G, Jain A, Kumar A et al (2020) Exosomes secreted by prostate cancer cells under hypoxia promote matrix metalloproteinases activity at pre-metastatic niches. Molecular carcinogenesis Mar 59(3):323-332. doi:10.1002/mc.23157

9. He L, Zhu W, Chen Q et al (2019) Ovarian cancer cell-secreted exosomal miR-205 promotes metastasis by inducing angiogenesis. Theranostics 9(26):8206-8220. doi:10.7150/thno.37455

10. Yokoi A, Yoshioka Y, Yamamoto $Y$ et al (2017) Malignant extracellular vesicles carrying MMP1 mRNA facilitate peritoneal dissemination in ovarian cancer. Nature communications Mar 6:8:14470. 
doi: $10.1038 /$ ncomms 14470

11. Tominaga N, Kosaka N, Ono M et al (2015) Brain metastatic cancer cells release microRNA-181ccontaining extracellular vesicles capable of destructing blood-brain barrier. Nature communications Apr 1:6:6716. doi:10.1038/ncomms7716

12. Wei F, Ma C, Zhou T et al (2017) Exosomes derived from gemcitabine-resistant cells transfer malignant phenotypic traits via delivery of miRNA-222-3p. Molecular cancer Jul 25(1):132. doi:10.1186/s12943-017-0694-8 16 ).

13. Naito Y, Yamamoto Y, Sakamoto $\mathrm{N}$ et al (2019) Cancer extracellular vesicles contribute to stromal heterogeneity by inducing chemokines in cancer-associated fibroblasts. Oncogene Jul 38(28):55665579. doi:10.1038/s41388-019-0832-4

14. Li DQ, Wang L, Fei F et al (2006) Identification of breast cancer metastasis-associated proteins in an isogenic tumor metastasis model using two-dimensional gel electrophoresis and liquid chromatography-ion trap-mass spectrometry. Proteomics Jun 6(11):3352-3368. doi:10.1002/pmic.200500617

15. Liu ZB, Hou YF, Di GH, Wu J, Shen ZZ, Shao ZM (2009) PA-MSHA inhibits proliferation and induces apoptosis through the up-regulation and activation of caspases in the human breast cancer cell lines. Journal of cellular biochemistry Sep 1(1):195-206. doi:10.1002/jcb.22241 108 ) .

16. Chang XZ, Li DQ, Hou YF et al (2008) Identification of the functional role of AF1Q in the progression of breast cancer. Breast cancer research treatment Sep 111(1):65-78. doi:10.1007/s10549-0079761-y

17. Shimoda M, Khokha R. Metalloproteinases in extracellular vesicles. Biochimica et biophysica acta Molecular cell research. Nov 2017;1864(11 Pt A):1989-2000. doi:10.1016/j.bbamcr.2017.05.027

18. Han KY, Dugas-Ford J, Seiki M, Chang JH, Azar DT. Evidence for the Involvement of MMP14 in MMP2 Processing and Recruitment in Exosomes of Corneal Fibroblasts. Investigative ophthalmology \& visual science. Aug 2015;56(9):5323-9. doi:10.1167/iovs.14-14417

19. Clancy JW, Sedgwick A, Rosse C et al (2015) Regulated delivery of molecular cargo to invasive tumour-derived microvesicles. Nature communications Apr 21:6:6919. doi:10.1038/ncomms7919

20. Ando W, Kikuchi K, Uematsu T et al (2019) Novel breast cancer screening: combined expression of miR-21 and MMP-1 in urinary exosomes detects $95 \%$ of breast cancer without metastasis. Scientific reports Sep 19(1):13595. doi:10.1038/s41598-019-50084-5 9 ) .

21. Zhang Y, Bi J, Huang J, Tang Y, Du S, Li P. Exosome (2020) A Review of Its Classification, Isolation Techniques, Storage, Diagnostic and Targeted Therapy Applications. Int J Nanomed 15:6917-6934. doi:10.2147/ijn.S264498

22. Doyle LM, Wang MZ. Overview of Extracellular Vesicles, Their Origin, Composition, Purpose, and Methods for Exosome Isolation and Analysis. Cells Jul 15 2019;8(7)doi:10.3390/cells8070727

23. Niland S, Eble JA. Hold on or Cut? Integrin- and MMP-Mediated Cell-Matrix Interactions in the Tumor Microenvironment. International journal of molecular sciences Dec 28 2020;22(1)doi:10.3390/ijms22010238 
24. Rabie EM, Zhang SX, Kourouklis AP et al (2021) Matrix degradation and cell proliferation are coupled to promote invasion and escape from an engineered human breast microtumor. Integrative biology: quantitative biosciences from nano to macro. Feb 3(1):17-29. doi:10.1093/intbio/zyaa026 13 ) .

25. Harati R, Hafezi S, Mabondzo A, Tlili A (2020) Silencing miR-202-3p increases MMP-1 and promotes a brain invasive phenotype in metastatic breast cancer cells. PloS one 15(10):e0239292. doi:10.1371/journal.pone.0239292

26. Wang QM, Lv L, Tang Y, Zhang L, Wang LF (2019) MMP-1 is overexpressed in triple-negative breast cancer tissues and the knockdown of MMP-1 expression inhibits tumor cell malignant behaviors in vitro. Oncology letters Feb 17(2):1732-1740. doi:10.3892/ol.2018.9779

27. Cui Q, Wang B, Li K et al. Upregulating MMP-1 in carcinoma-associated fibroblasts reduces the efficacy of Taxotere on breast cancer synergized by Collagen IV. Oncology letters. Sep 2018;16(3):3537-3544. doi:10.3892/ol.2018.9092

28. Goerge T, Barg A, Schnaeker EM et al (2006) Tumor-derived matrix metalloproteinase-1 targets endothelial proteinase-activated receptor 1 promoting endothelial cell activation. Cancer research Aug 1(15):7766-7774. doi:10.1158/0008-5472.CAN-05-3897 66 ) .

29. Boire A, Covic L, Agarwal A, Jacques S, Sherifi S, Kuliopulos A (2005) PAR1 is a matrix metalloprotease-1 receptor that promotes invasion and tumorigenesis of breast cancer cells. Cell Feb 11(3):303-313. doi:10.1016/j.cell.2004.12.018 120 ) .

30. Juncker-Jensen A, Deryugina El, Rimann I et al (2013) Tumor MMP-1 activates endothelial PAR1 to facilitate vascular intravasation and metastatic dissemination. Cancer research Jul 15(14):41964211. doi:10.1158/0008-5472.Can-12-4495 73 ) .

31. Tekin C, Aberson HL, Waasdorp C et al (2020) Macrophage-secreted MMP9 induces mesenchymal transition in pancreatic cancer cells via PAR1 activation. Cellular oncology (Dordrecht) Dec 43(6):1161-1174. doi:10.1007/s13402-020-00549-x

32. Covic L, Kuliopulos A. Protease-Activated Receptor 1 as Therapeutic Target in Breast, Lung, and Ovarian Cancer: Pepducin Approach. International journal of molecular sciences Jul 31 2018;19(8)doi:10.3390/ijms19082237

33. Scheau C, Badarau IA, Costache R et al (2019) The Role of Matrix Metalloproteinases in the Epithelial-Mesenchymal Transition of Hepatocellular Carcinoma. Analytical cellular pathology (Amsterdam) 2019:9423907. doi:10.1155/2019/9423907

34. Wang W, Li X, Zhang W et al (2014) Oxidored-nitro domain containing protein 1 (NOR1) expression suppresses slug/vimentin but not snail in nasopharyngeal carcinoma: Inhibition of EMT in vitro and in vivo in mice. Cancer letters Jun 28(1-2):109-118. doi:10.1016/j.canlet.2014.03.005 348 ) .

35. Shimoda M (2019) Extracellular vesicle-associated MMPs: A modulator of the tissue microenvironment. Adv Clin Chem 88:35-66. doi:10.1016/bs.acc.2018.10.006

36. Zuo LL, Zhang J, Liu LZ et al (2017) Cadherin 6 is activated by Epstein-Barr virus LMP1 to mediate EMT and metastasis as an interplay node of multiple pathways in nasopharyngeal carcinoma. Oncogenesis Dec 22(12):402. doi:10.1038/s41389-017-0005-7 6 ) . 
37. Li Z, Jiang P, Li J et al (2018) Tumor-derived exosomal Inc-Sox2ot promotes EMT and stemness by acting as a ceRNA in pancreatic ductal adenocarcinoma. Oncogene Jul 37(28):3822-3838. doi:10.1038/s41388-018-0237-9

38. Eisenhauer EA, Therasse $\mathrm{P}$, Bogaerts $\mathrm{J}$ et al. New response evaluation criteria in solid tumours: revised RECIST guideline (version 1.1). European journal of cancer (Oxford, England: 1990). Jan 2009;45(2):228 - 47. doi:10.1016/j.ejca.2008.10.026

39. Kosaka N, Yoshioka Y, Fujita Y, Ochiya T (2016) Versatile roles of extracellular vesicles in cancer. The Journal of clinical investigation Apr 1 126(4):1163-1172. doi:10.1172/jci81130

40. Costa-Silva B, Aiello NM, Ocean AJ et al (2015) Pancreatic cancer exosomes initiate pre-metastatic niche formation in the liver. Nature cell biology Jun 17(6):816-826. doi:10.1038/ncb3169

41. Fatima F, Nawaz M. Vesiculated Long Non-Coding RNAs: Offshore Packages Deciphering TransRegulation between Cells, Cancer Progression and Resistance to Therapies. Non-coding RNA. Feb 23 2017;3(1)doi:10.3390/ncrna3010010

42. Maia J, Caja S, Strano Moraes MC, Couto N, Costa-Silva B (2018) Exosome-Based Cell-Cell Communication in the Tumor Microenvironment. Frontiers in cell developmental biology 6:18. doi:10.3389/fcell.2018.00018

43. Peinado H, Lavotshkin S, Lyden D (2011) The secreted factors responsible for pre-metastatic niche formation: old sayings and new thoughts. Seminars in cancer biology Apr 21(2):139-146. doi:10.1016/j.semcancer.2011.01.002

44. Li K, Chen Y, Li A, Tan C, Liu X (2019) Exosomes play roles in sequential processes of tumor metastasis. International journal of cancer Apr 1(7):1486-1495. doi:10.1002/ijc.31774 144 ) .

45. Kalluri R, LeBleu VS. The biology, function, and biomedical applications of exosomes. Science (New York, NY). Feb 7 2020;367(6478)doi:10.1126/science.aau6977

46. Wang K, Zheng J, Yu J et al (2020) Knockdown of MMP-1 inhibits the progression of colorectal cancer by suppressing the PI3K/Akt/c-myc signaling pathway and EMT. Oncology reports Apr 43(4):1103-1112. doi:10.3892/or.2020.7490

47. Li H, Qiu Z, Li F, Wang C (2017) The relationship between MMP-2 and MMP-9 expression levels with breast cancer incidence and prognosis. Oncology letters Nov 14(5):5865-5870. doi:10.3892/ol.2017.6924

48. Radisky ES, Raeeszadeh-Sarmazdeh M, Radisky DC (2017) Therapeutic Potential of Matrix Metalloproteinase Inhibition in Breast Cancer. Journal of cellular biochemistry Nov 118(11):35313548. doi:10.1002/jcb.26185

\section{Tables}

Table 1. Patient Characteristics at baseline $(N=134)$ 


\begin{tabular}{|ll|}
\hline Characteristics & Pts (N=134) \\
\hline Age (Median, range) & $52,24-82$ \\
\hline$<40$ years & $18(13.43 \%)$ \\
\hline$\geq 40$ years & $116(86.56 \%)$ \\
\hline Menstruation status & \\
\hline Post-menopausal & $86(64.18 \%)$ \\
\hline Pre-menopausal & $48(35.82 \%)$ \\
\hline Pathological tumor size & \\
\hline$\leq 2$ cm & $32(23.88 \%)$ \\
\hline $2-5 \mathrm{~cm}$ & $97(72.39 \%)$ \\
\hline$>5 \mathrm{~cm}$ & $5(3.73 \%)$ \\
\hline Grade & \\
\hline$\square$ & \\
\hline$\square$ & \\
\hline
\end{tabular}

Page 18/28 


\begin{tabular}{|ll|}
\hline Nodal status & \\
\hline O positive nodes & $74(55.22 \%)$ \\
\hline $1-3$ positive nodes & $40(29.85 \%)$ \\
\hline$\geq 4$ positive nodes & $20(1.54 \%)$ \\
\hline Prior Adjuvant Chemotherapy & \\
\hline Anthracyclines & $3(2.24 \%)$ \\
\hline Taxanes & $15(11.19 \%)$ \\
\hline Both & $88(65.67 \%)$ \\
\hline Unknown & $28(20.90 \%)$ \\
\hline
\end{tabular}

Table 2 Patient Characteristics at baseline $(N=48)$ 


\begin{tabular}{|c|c|c|}
\hline Characteristics & $\begin{array}{l}\text { Initial metastasis } \\
(\mathrm{N}=30)\end{array}$ & $\begin{array}{l}\text { Post-operation } \\
(\mathrm{N}=18)\end{array}$ \\
\hline & $\mathrm{N}(\%)$ & $\mathrm{N}(\% 区$ \\
\hline Age (Median, range) & $50 \rrbracket 31-66$ & $51,31-68$ \\
\hline$<40$ years & $7(23.3 \%)$ & $3(16.7 \%)$ \\
\hline$\geq 40$ years & $23(76.7 \%)$ & $15(83.3 \%)$ \\
\hline \multicolumn{3}{|l|}{ Menstruation status } \\
\hline Post-menopausal & $20(66.7 \%)$ & $5(27.8 \%)$ \\
\hline Pre-menopausal & $10(33.3 \%)$ & $13(72.2 \%)$ \\
\hline \multicolumn{3}{|c|}{ ECOG performance status } \\
\hline 0 & $0(0)$ & $0(0)$ \\
\hline$\geq 1$ & $30(100 \%)$ & $18(100 \%)$ \\
\hline \multicolumn{3}{|c|}{ Neoadjuvant Chemotherapy } \\
\hline Yes & $3(10 \%)$ & $0(0)$ \\
\hline No & $27(90 \%)$ & $18(100 \%)$ \\
\hline \multicolumn{3}{|c|}{ Pathological tumor size } \\
\hline 0 to $\leq 2 \mathrm{~cm}$ & $14(46.7 \%)$ & $12(66.7 \%)$ \\
\hline 2 to $\leq 5 \mathrm{~cm}$ & $13(43.4 \%)$ & $5(27.8 \%)$ \\
\hline$>5 \mathrm{~cm}$ & $3(10 \%)$ & $1(5.56 \%)$ \\
\hline \multicolumn{3}{|c|}{ Number of metastatic sites } \\
\hline$<3$ & $17(56.7 \%)$ & - \\
\hline$\geq 3$ & $13(43.3 \%)$ & - \\
\hline \multicolumn{3}{|l|}{ Metastatic sites } \\
\hline Lymph nodes & $19(63.3 \%)$ & - \\
\hline Liver & $3(10 \%)$ & - \\
\hline Bone & $9(30 \%)$ & - \\
\hline Lung & $18(60 \%)$ & - \\
\hline Chest wall & $6(20 \%)$ & - \\
\hline Pleura & $2(6.67 \%)$ & - \\
\hline
\end{tabular}




\begin{tabular}{|lll|}
\hline Others & $0(0)$ & - \\
\hline Visceral metastasis & & \\
Yes & $24(80 \%)$ & - \\
\hline No & $6(20)$ & - \\
\hline Disease-free survival & & - \\
$>12$ months & $26(86.7 \%)$ & - \\
$\leq 12$ months & $4(13.3 \%)$ & - \\
\hline
\end{tabular}

ECOG, Eastern Cooperative Oncology Group;

\section{Figures}


A

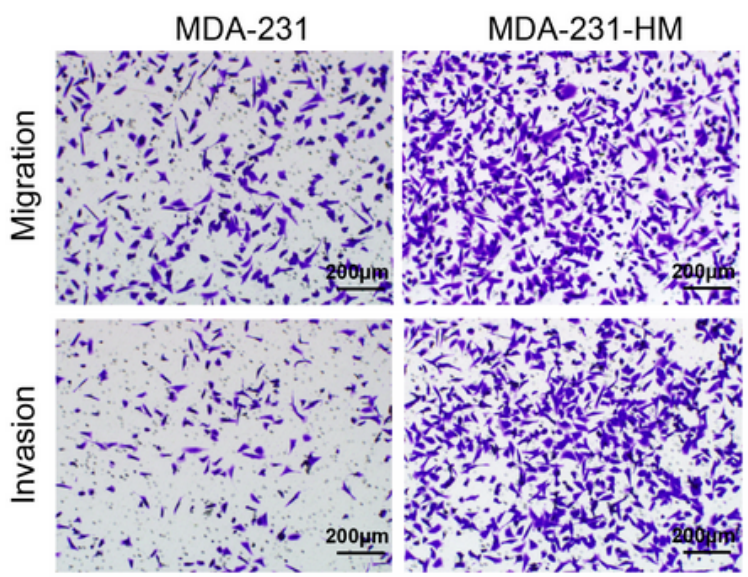

B

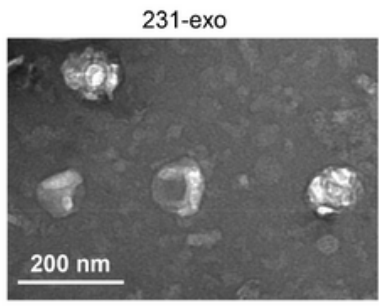

D
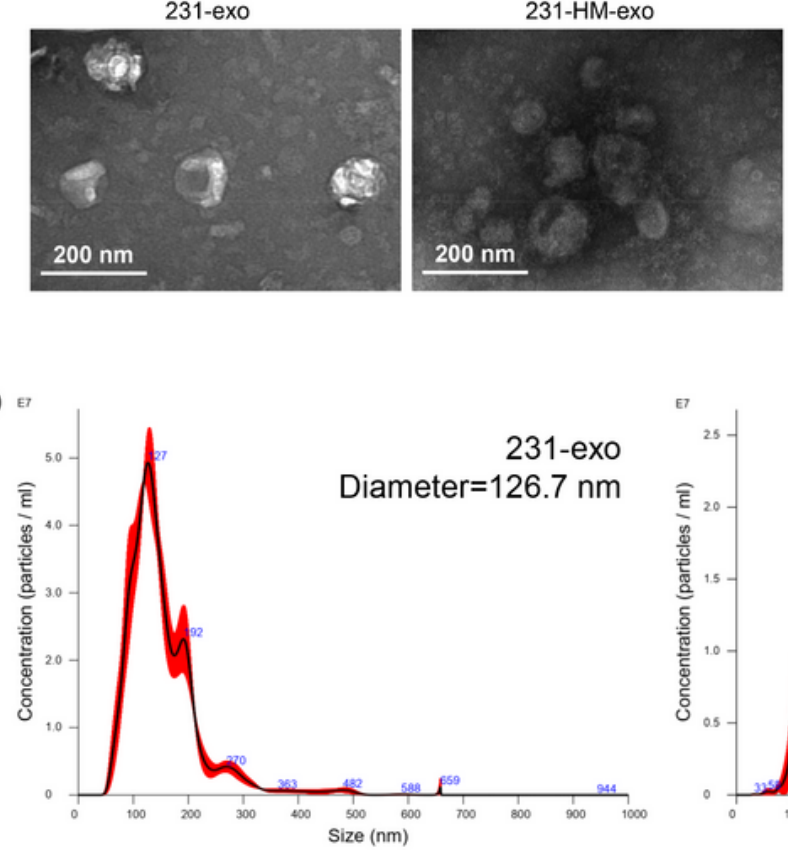

C
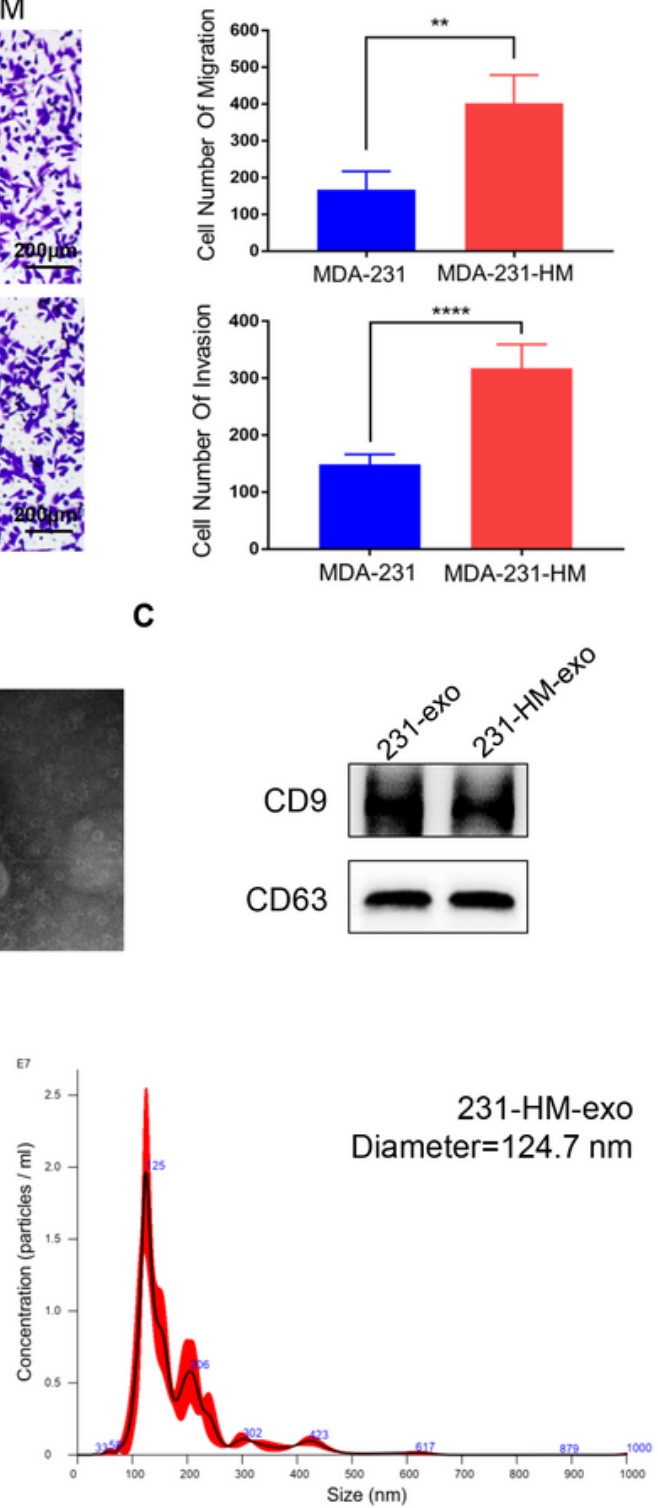

E

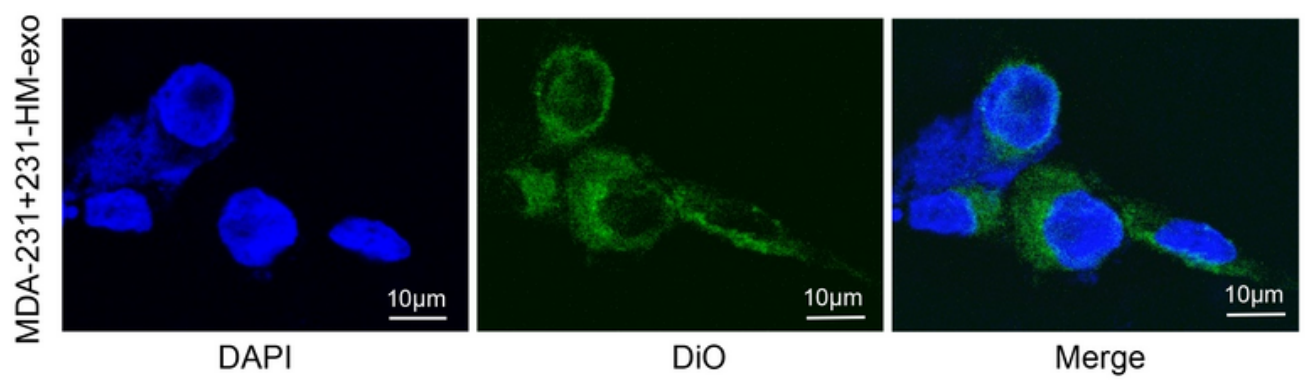

Figure 1

Isolation and verification of exosomes. (A). Migration and invasion assay of MDA-231 cells and MDA231-HM cells. Scale bar: 200 um. (B). Transmission electron microscopy image of 231-exo and 231-HMexo. Scale bar: $100 \mathrm{~nm}$. (C). Western blot analysis of exosome markers in 231-exo and 231-HM-exo. (D). Nanoparticle analysis of 231-exo and 231-HM-exo, confirming the expected size range of 30-150 nm in diameter. (E). Immunofluorescence images of DiO-labeled 231-HM-exo (green) ingested by MDA-231 cells 
(blue) after co-culture for $24 \mathrm{~h}$. Scale bar: $10 \mu \mathrm{m}$. Representative images were presented from three independent experiments. Data are shown as the mean \pm SD. N.S., no significance, ${ }^{*} p<0.05,{ }^{*} p<0.01$.

A

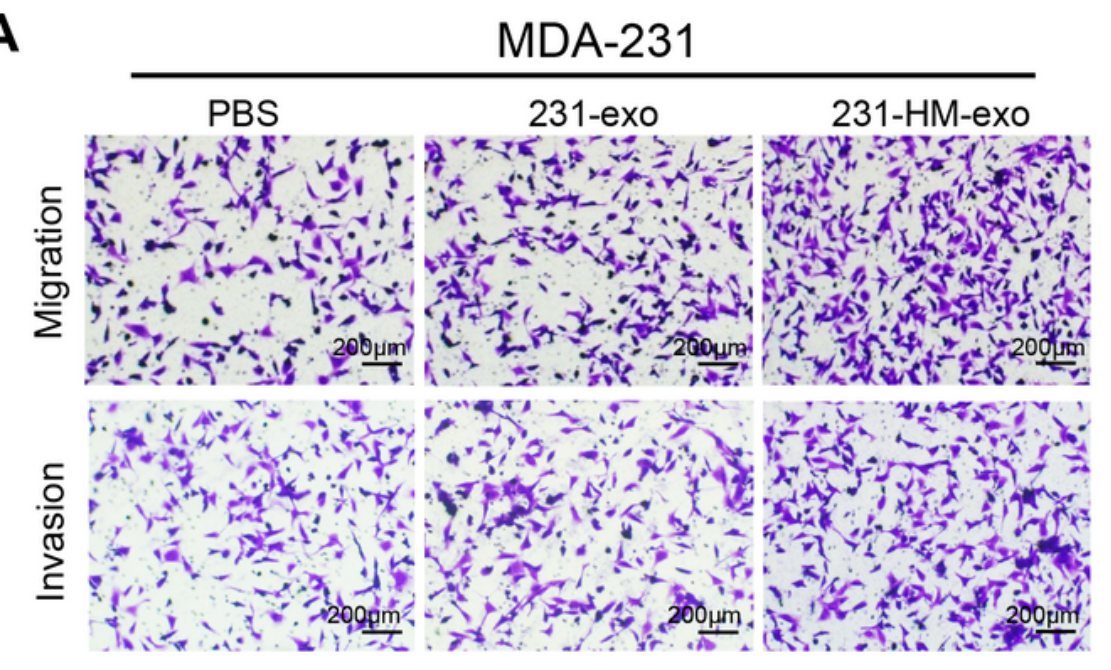

B

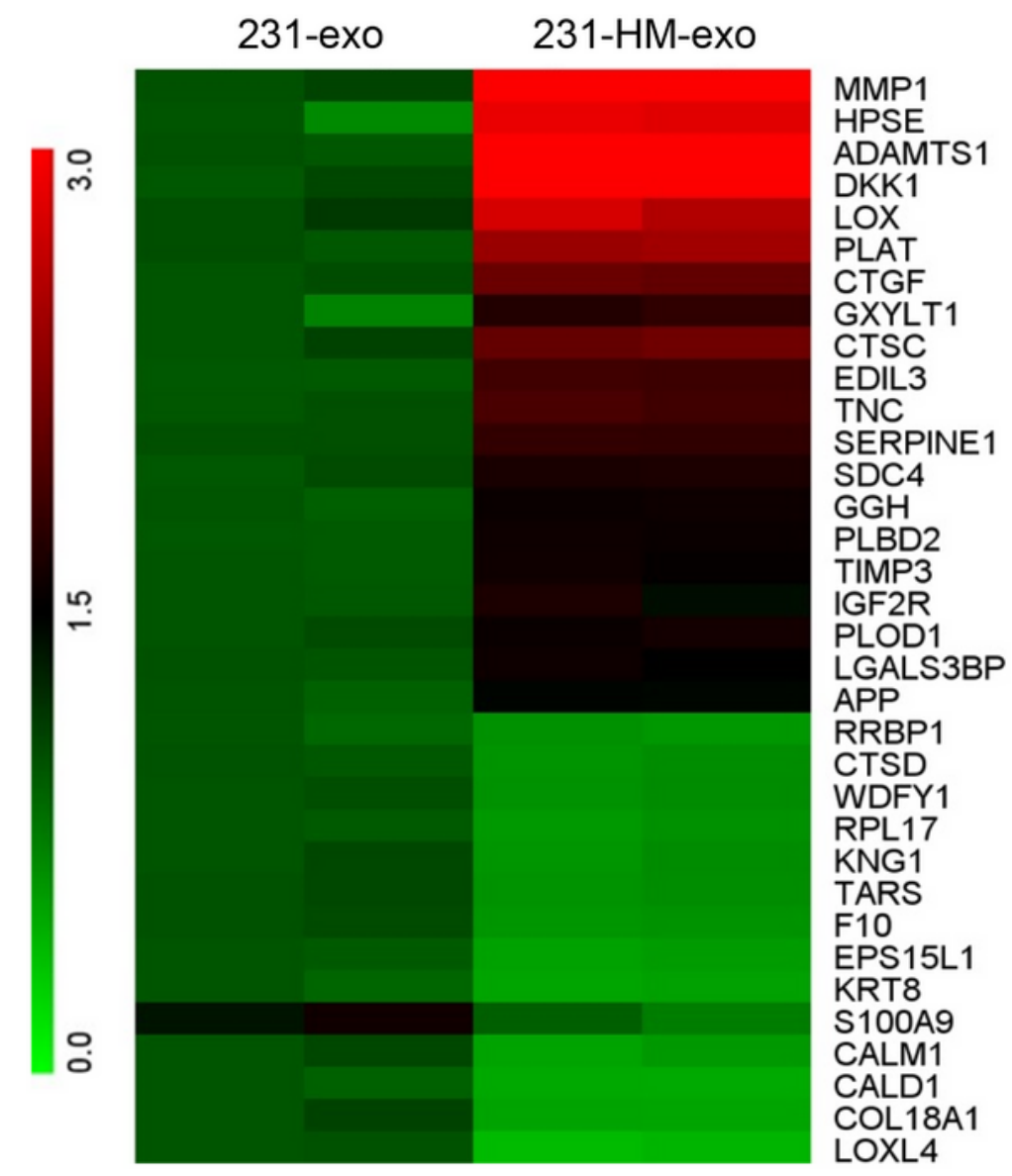

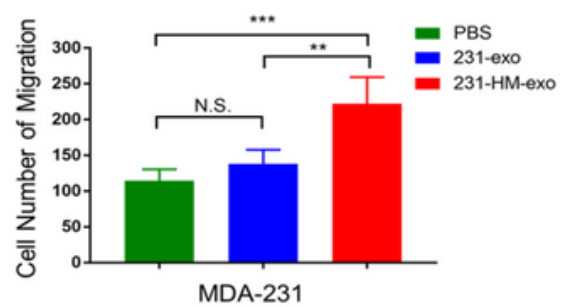

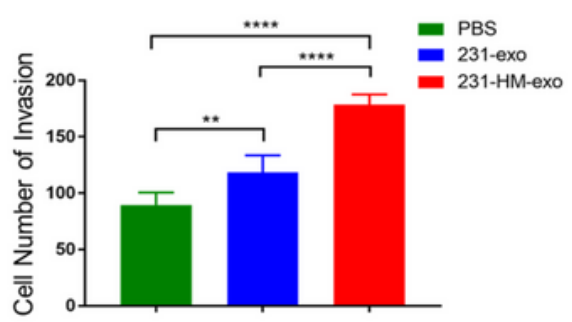

MDA-231

C
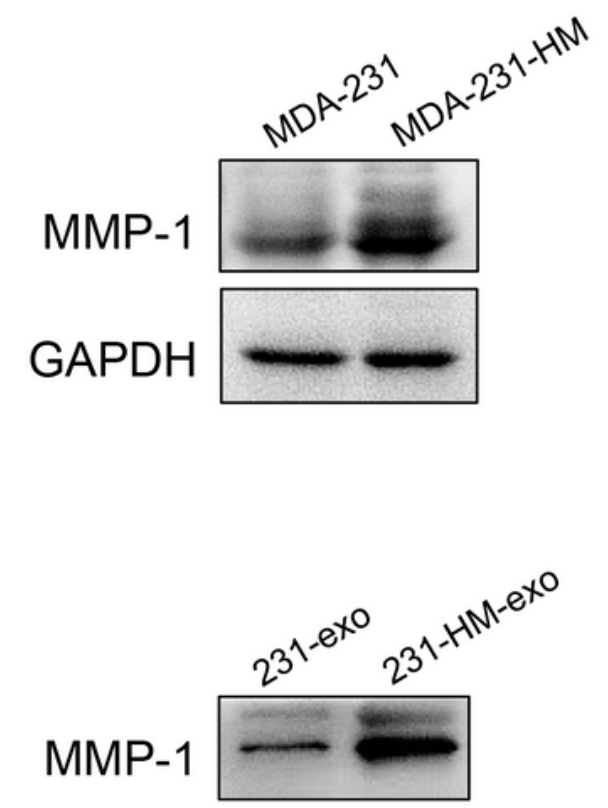

CD63

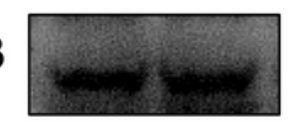

\section{Figure 2}

MMP-1 was upregulated in 231-HM-exo. (A). Transwell assay of MDA-231 cells pretreated with PBS, 231exo and 231-HM-exo. Scale bar: $200 \mu \mathrm{m}$. (B). Heat map showing the relative expression of proteins in 231exo and 231-HM-exo. (C). Western blot analysis of MMP-1 in MDA-231, MDA-231-HM cells, 231-exo and 
231-HM-exo. Representative images were presented from three independent experiments. Data are shown as the mean \pm SD. N.S., no significance, ${ }^{*} p<0.05,{ }^{*} p<0.01$.

A

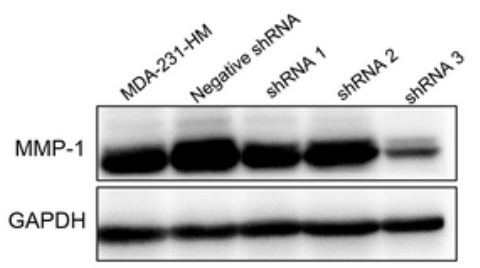

C

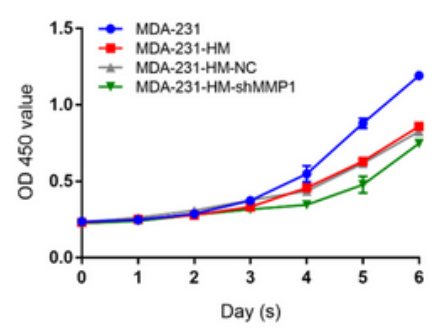

D
B
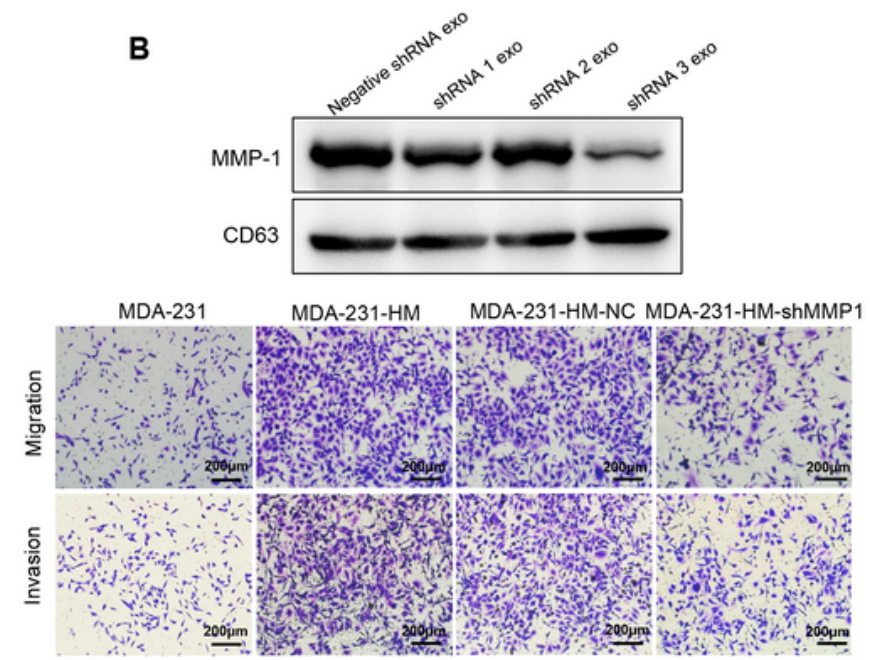

E
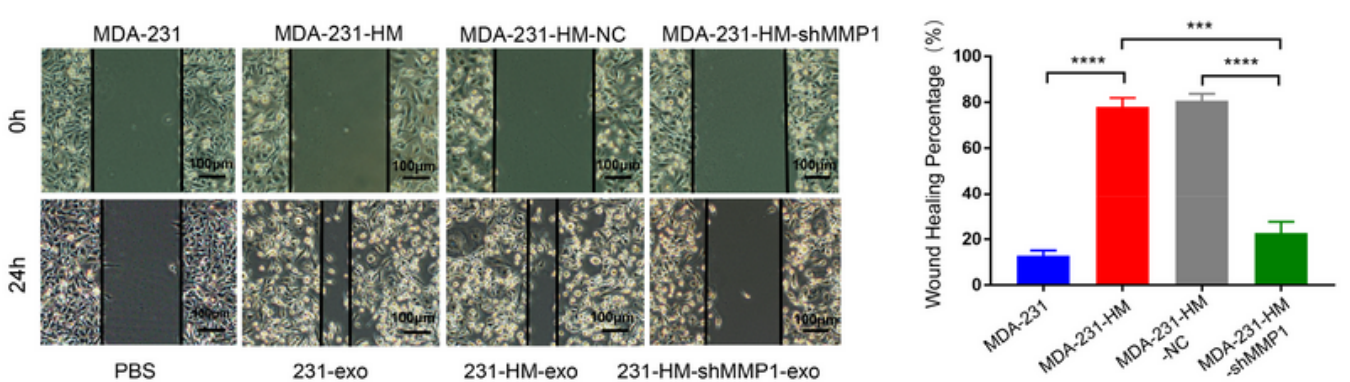

$\mathbf{F}$

PBS

231-exo

231-HM-exo 231-HM-shMMP1-exo
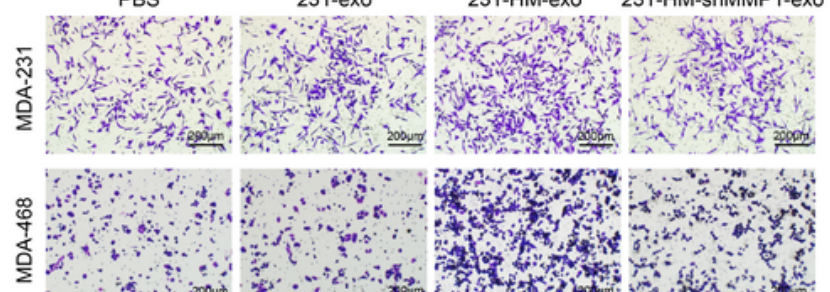

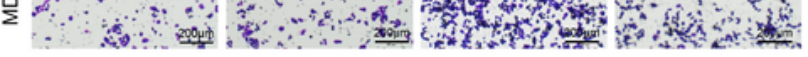
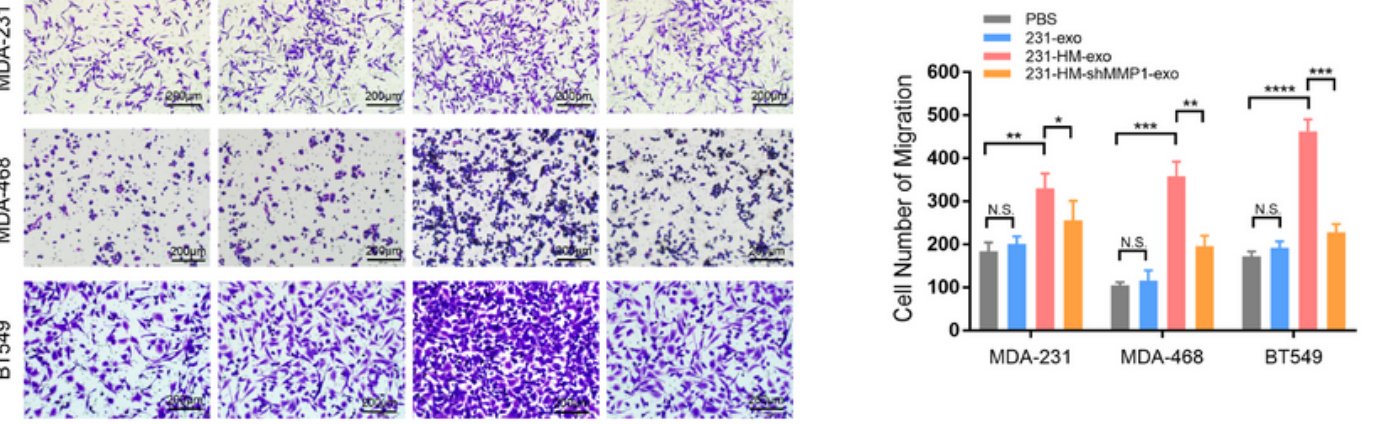

G
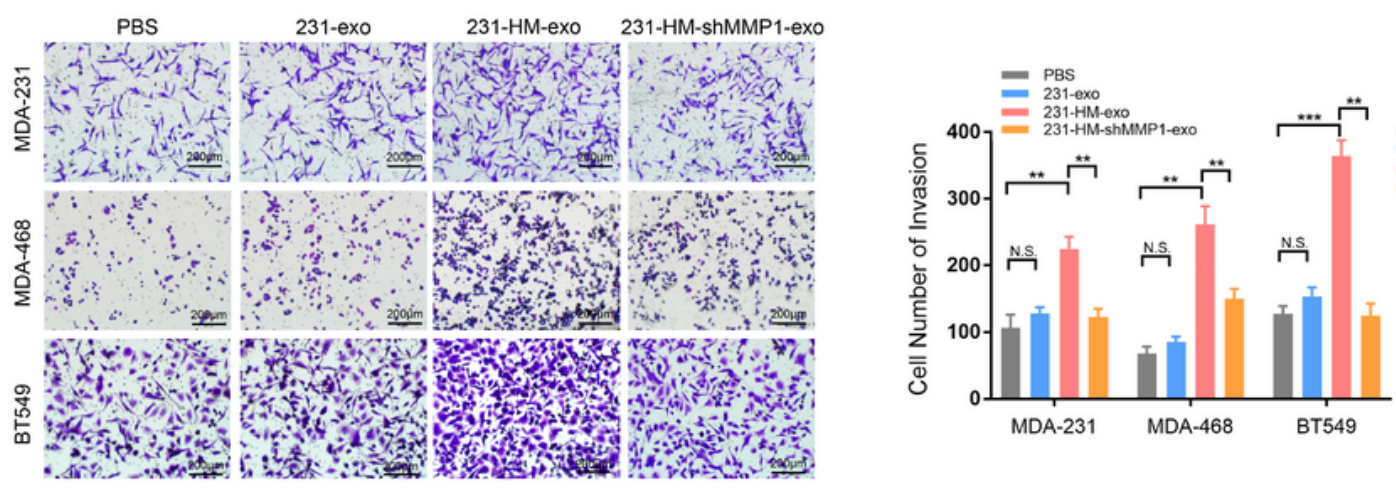

Figure 3

Targeted knockdown of MMP-1 RNA inhibits exosomes-mediated metastasis in vitro. (A). Downregulation of MMP-1 in MDA-231-HM cells by short-heparin RNA. (B). Western blot analysis of MMP-1 in exosomes purified from MMP-1-shRNA-treated 231 HM cells. (C). CCK-8 assay of MDA-231, MDA-231-HM, MDA-231- 
HM-NC and MDA-231-HM-shMMP1 cells. (D). Transwell migration and invasion assay of MDA-231, MDA-

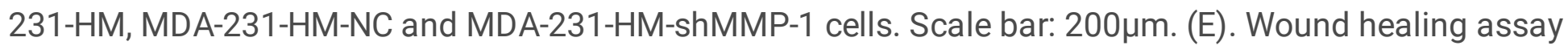

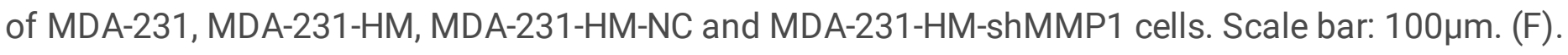
Migration assay of MDA-231, MDA-468 and BT549 cells co-cultured with PBS, 231-HM-exo, 231-HM-NCexo and 231HM-shMMP-1 for 24h. Scale bar: $200 \mu \mathrm{m}$. (G). Invasion assay of MDA-231, MDA-468 and BT549 cells co-cultured with PBS, 231-HM-exo, 231-HM-NC-exo and 231HM-shMMP1-exo for 24h. Scale bar: $200 \mu \mathrm{m}$.Data are shown as the mean \pm SD. N.S., no significance, ${ }^{\star} \mathrm{p}<0.05,{ }^{\star \star} \mathrm{p}<0.01,{ }^{\star \star *} \mathrm{p} \otimes 0.005$, $\star \star \star \star p<0.001$. 
A

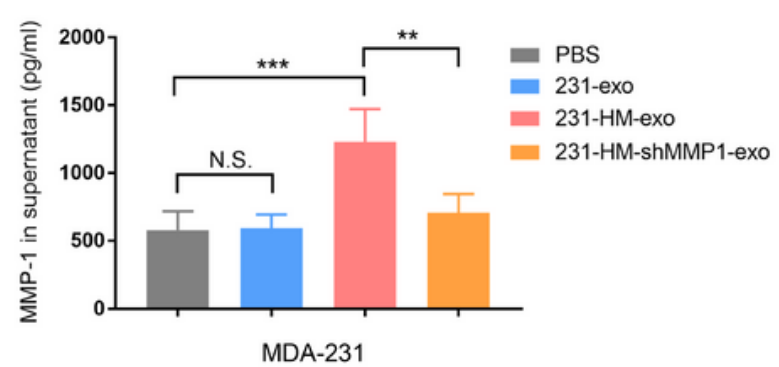

B

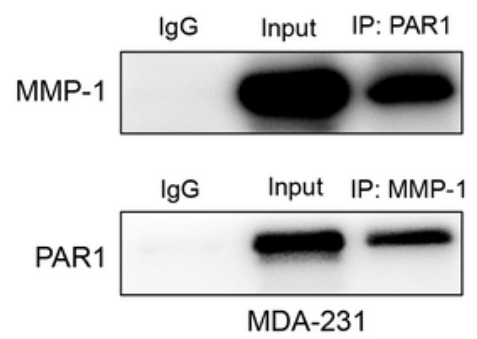

C
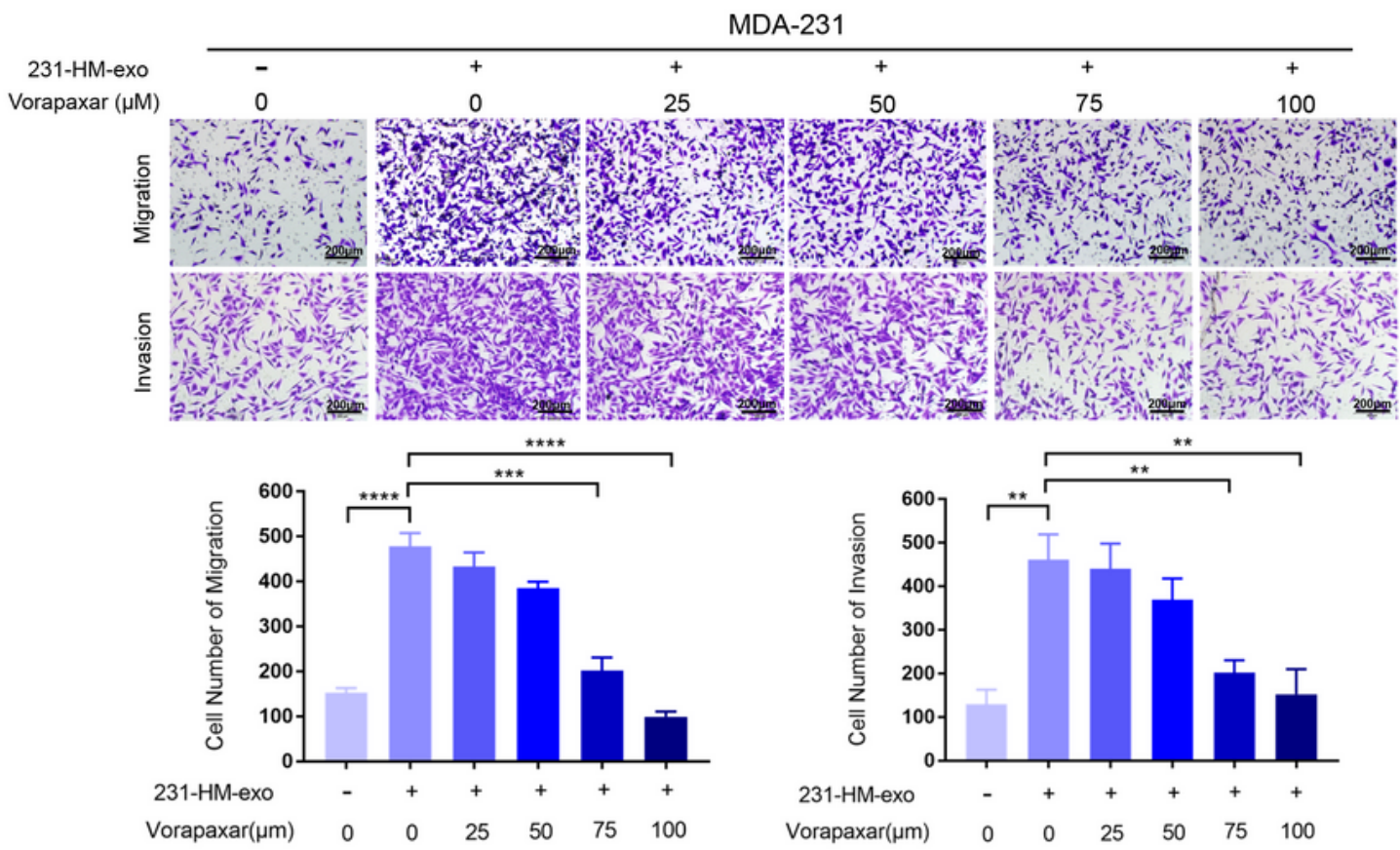

D

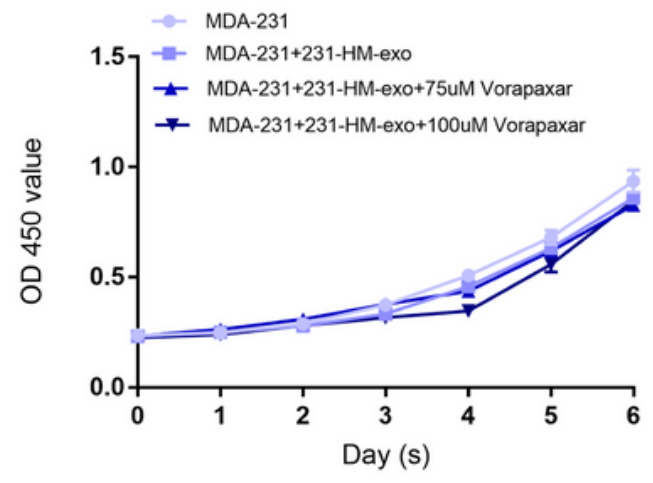

E

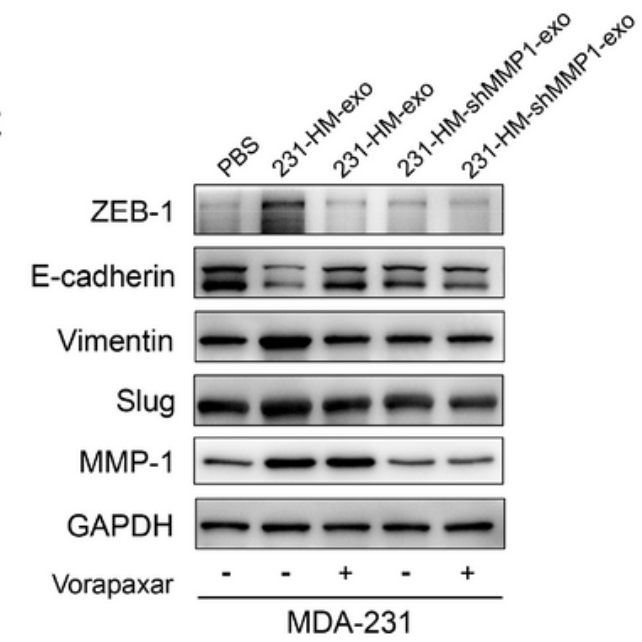

\section{Figure 4}

MMP-1 binding to PAR1 promotes the metastasis of TNBC possibly through EMT. (A). Quantification of MMP-1 in supernatants of 231 cells after pre-treated with PBS, 231-exo, 231-HM-exo and 231-HMshMMP1-exo, measured by ELISA. (B). Whole-cell lysates from activated 231 cells were subjected to immunoprecipitation with anti-MMP-1 and immunoblotted for PAR1, then with anti-PAR1 and immunoblotted for MMP-1. (C). Transwell migration and invasion assay of MDA-231 cells treated with 


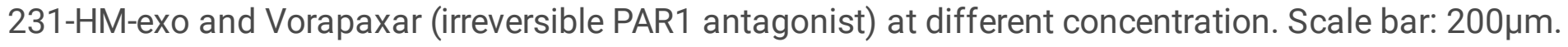
(D). CCK-8 assay of MDA-231 cells co-cultured with 231-HM-exo and Vorapaxar at certain concentration. (E). Western blot analysis of MMP-1 and EMT-related biomarkers in MDA-231 cells co-cultured with PBS, 231-exo, 231-HM-exo and 231-HM-shMMP1-exo. Representative images were presented from three independent experiments. Data are shown as the mean \pm SD. ${ }^{*} p<0.05,{ }^{\star \star} p<0.01,{ }^{\star \star \star} \mathrm{p}<0.005,{ }^{\star \star \star \star} \mathrm{p}<$ 0.001 .

A
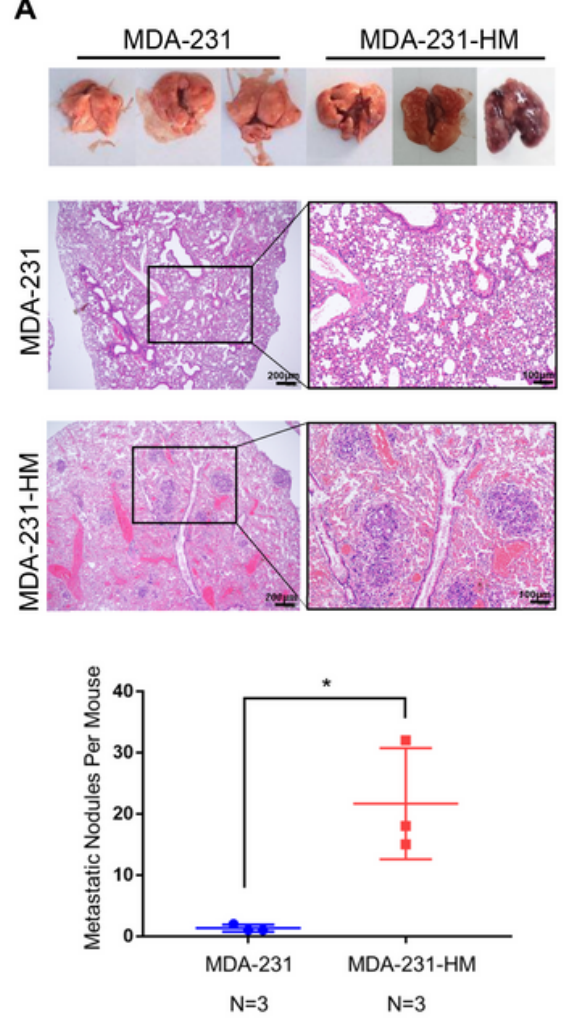

B

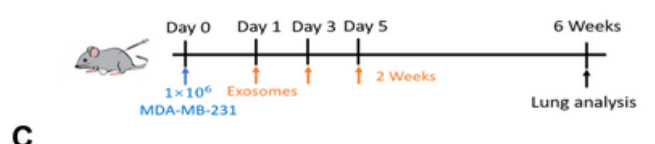

C

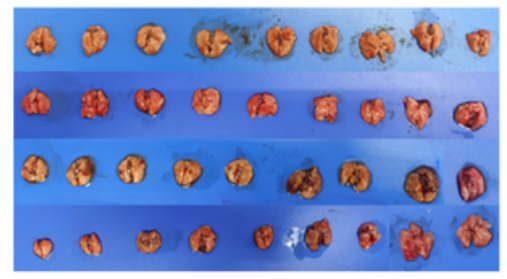

D

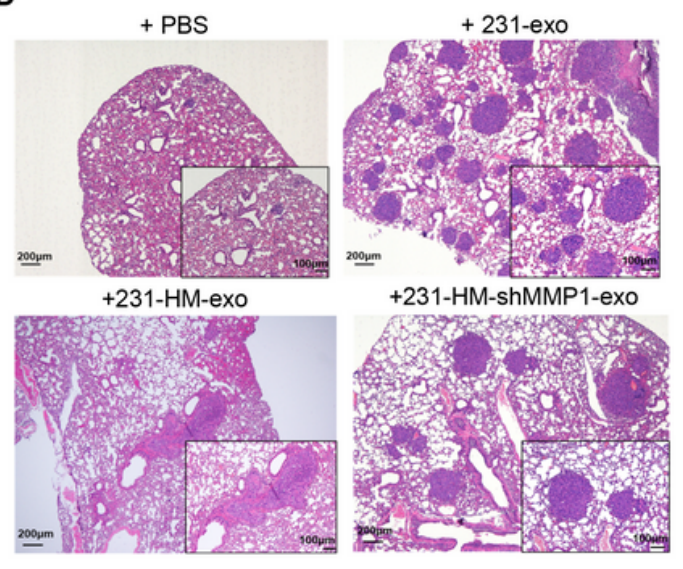

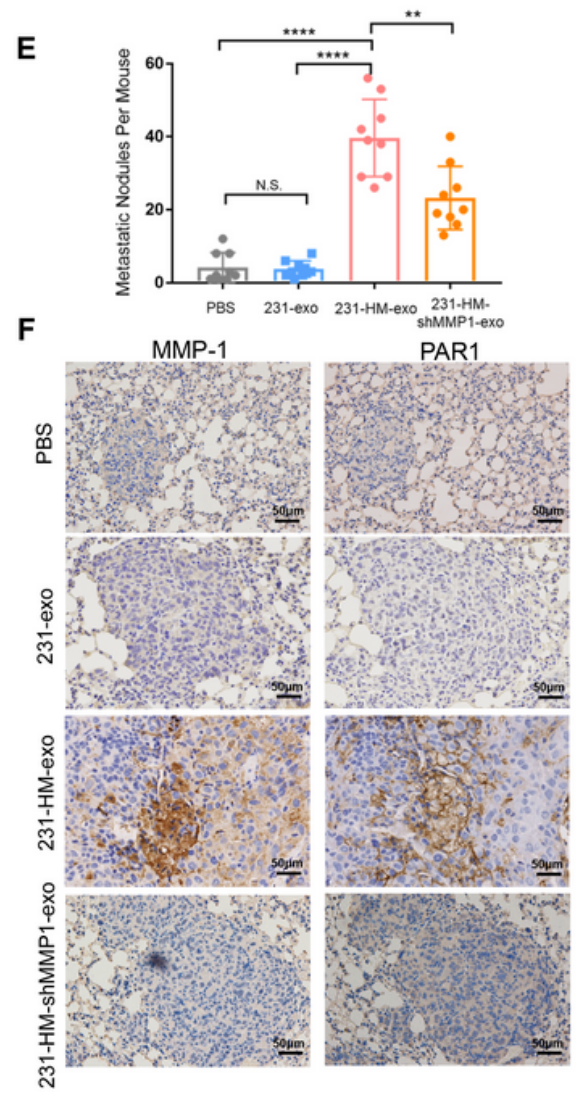

\section{Figure 5}

Exosomal MMP-1 mediates the transmission of metastasis capability in vivo. (A). Representative lung tissues and H\&E images from lung sections of mice injected with $1 \times 106231$ and $231 \mathrm{HM}$ cells in the tail vein ( $n=3$ in each group). Scale bar: $200 \mu m$ (left), $100 \mu m$ (right). Number of metastatic lung nodules per mouse as determined by sum of three coronal sections per mouse. (B). Schematic diagram of lung metastasis model in nude mice. (C-D). Representative lung tissues(C) and H\&E images(D) from lung sections of mice injected with $1 \times 106$ MDA-231 cells and then treated with PBS, 231-exo, 231-HM-exo and 231-HM-shMMP1-exo ( $\mathrm{n}=9$ in each group). (E). Number of metastatic lung nodules per mouse as determined by sum of three coronal sections per mouse. (F). Representative images for immunohistochemistry of MMP-1 and PAR1 in lung sections of nude mouse lung metastasis model. Scale bar: $50 \mu \mathrm{m}$. Representative images were presented from three independent experiments. Data are shown as the mean \pm SD. ${ }^{*} p<0.05,{ }^{* \star} p<0.01$, ${ }^{\star \star *} p \otimes 0.005$, ${ }^{\star \star * \star} p<0.001$. 
A

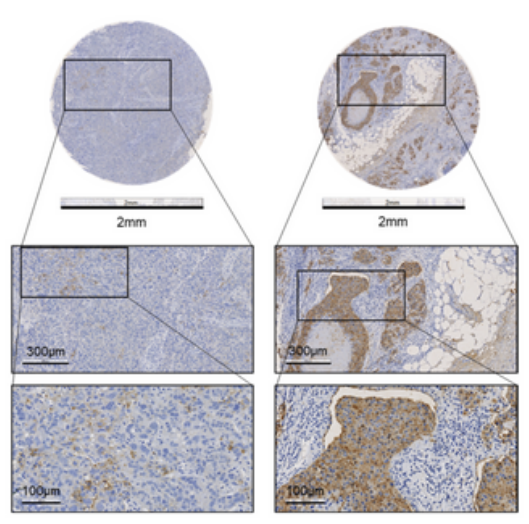

D

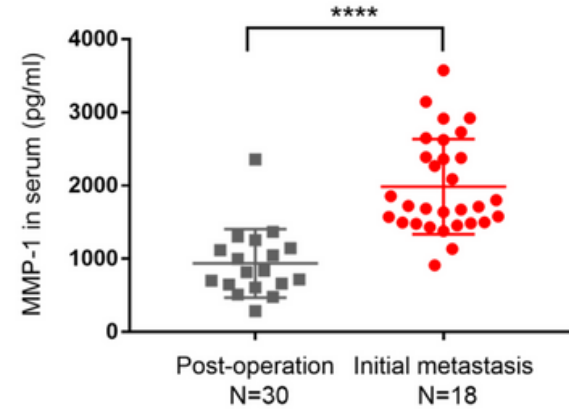

$\mathbf{F}$

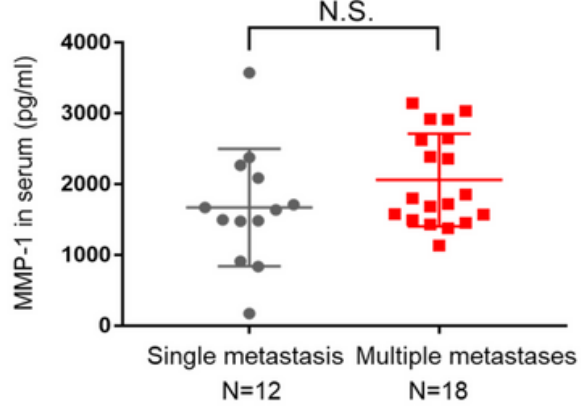

B

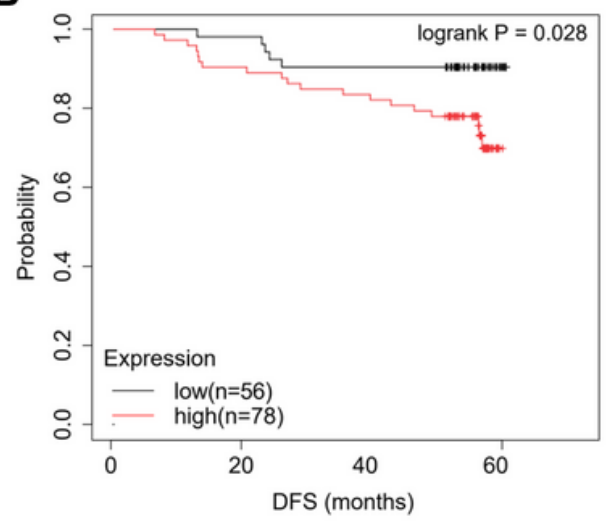

E

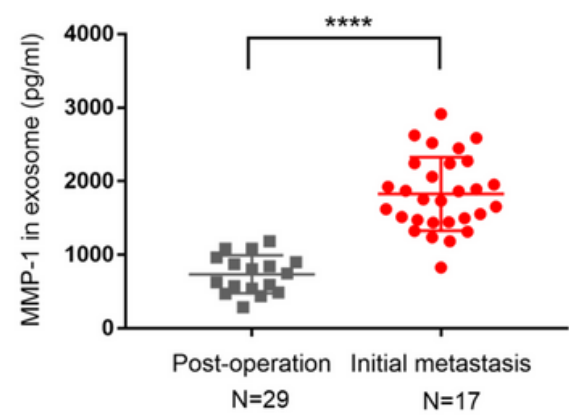

G

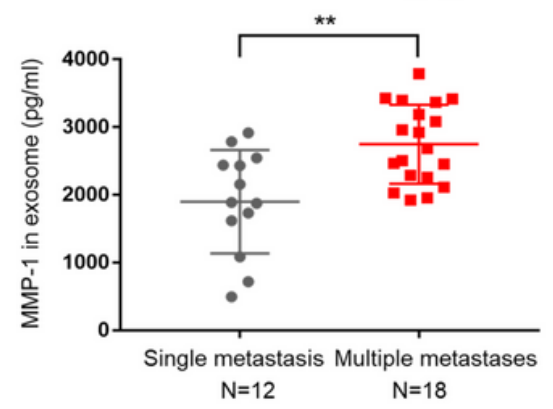

C

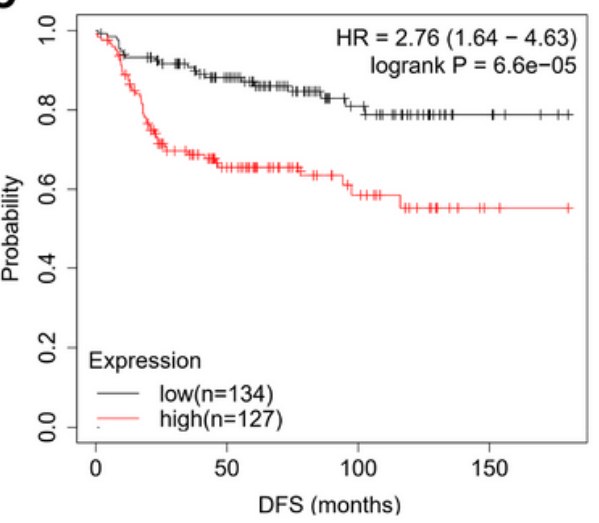

H
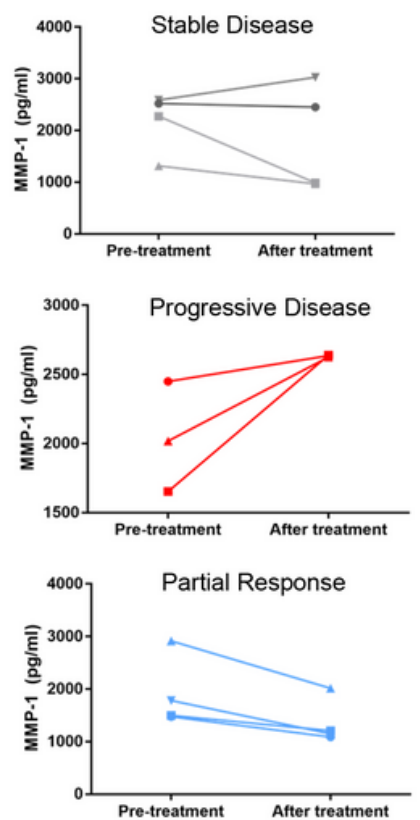

\section{Figure 6}

Plasma exosomal MMP-1 level is elevated in patients with shorter DFS. (A). Representative IHC stained images of breast cancer tissues of patients in Shanghai Cancer Center from Aug 2015 to Aug 2020 $(n=134)$, referring high $(n=78)$ and low $(n=56)$ expression of MMP-1. (B). Kaplan-Meier survival analysis showing the relationship between MMP-1 expression and DFS of breast cancer patients $(n=134)$. (C). The DFS curves of TNBC patients with high $(n=134)$ and low $(n=127)$ MMP-1 expression. Data was retrieved from the Kaplan Meier plotter (http://kmplot.com/analysis/). (D-E). Quantification of MMP-1 in serum and exosomes isolated from the serum of breast cancer patients before surgery $(n=18)$ and first diagnosed as metastasis $(n=30)$, measured by ELISA. $(F-G)$. Quantification of MMP-1 in serum and exosomes isolated from the serum of breast cancer patients with single metastatic site $(n=13)$ and multiple metastatic sites $(n=17)$, measured by ELISA. $(H)$. Quantification of MMP-1 in exosomes isolated from the serum of breast cancer patients $(n=11)$ before and after first-line chemotherapy, measured by ELISA. Efficacy has been assessed as 'stable disease' $(n=4)$, 'progressive disease' $(n=3)$ and 'partial response' $(n=4)$, respectively. Data are shown as the mean \pm SD. N.S., no significance, ${ }^{\star} p<0.05,{ }^{*} p<0.01$, $\star \star \star \mathrm{p} \otimes 0.005, * \star \star \star \mathrm{p}<0.001$. 